\title{
Analysis and Numerical Simulation on the Reduction Effect of Stress Waves Caused by Water Jet Slotting Near Blasting Source
}

\author{
Dengfeng Su, ${ }^{1}$ Yong Kang, ${ }^{1,2}$ Dongyang Li, ${ }^{1}$ Xiaochuan Wang, ${ }^{1,2}$ and Fuwen Yan ${ }^{1}$ \\ ${ }^{1}$ State Key Laboratory of Coal Mine Disaster Dynamics and Control, Chongqing University, Chongqing 400044, China \\ ${ }^{2}$ Hubei Key Laboratory of Waterjet Theory and New Technology, Wuhan University, Wuhan 430072, China \\ Correspondence should be addressed to Yong Kang; kangyong@whu.edu.cn
}

Received 4 November 2015; Revised 31 March 2016; Accepted 21 April 2016

Academic Editor: Shimin Liu

Copyright (C) 2016 Dengfeng Su et al. This is an open access article distributed under the Creative Commons Attribution License, which permits unrestricted use, distribution, and reproduction in any medium, provided the original work is properly cited.

As one of the most serious "side effects" of blast excavation, blast-induced vibration must be controlled for existing buildings and human beings. This paper proposes a method for blast-induced vibration reduction with water jet assistance according to the cutting characters of low-noised, environment-friendly water jet. The mechanism of vibration-isolation with water jet assistance was analyzed, and the stress wave energy attenuation models were established based on blasting theory and stress wave theory. Influence law on shock wave attenuation by vibration-isolation slot was studied by numerical simulation. Simulation results agree with the theoretical analysis roughly. The results of this study put forward a method for blast-induced vibration near blasting source and provide a certain theoretical basis.

\section{Introduction}

With the development of China's economy, the demand for energy and space is increasingly rapid. Therefore, a growing number of large underground space projects have been put into construction. As the most popular method for rock excavation, drilling-blasting method is still an indispensable means in engineering construction. However, it has been proved that only $20 \sim 30 \%$ of energy released by explosives is used for rock fragmentation during blasting; the rest appears in the form of ground vibration, air blast, fly rock, noise, and back break [1-3]. Among all the "side effects" of blasting, ground vibration, posing a serious threat to existing building structures, is a major concern to designers and environmentalists [4-6]. So it is very important and meaningful to reduce blast-induced vibration.

Generally, the methods for reducing blast-induced vibration include the following ones. The first solution is to adopt millisecond blasting or to decrease explosive charge per delay. Millisecond blasting technique has been verified as a promising strategy in mitigating the PPV level and a great number of analysis models and methods for blast signals have been carried out [7-10]. The method for decreasing explosive charge per delay may also affect particle size after blasting or production rate $[11,12]$. Adding sacrificial cladding in the borehole is the second solution. Guruprasad and Mukherjee [13] proposed layered mild steel plates employed to dissipate or absorb blast energy, and the impulse distributions and energy absorptions in plastic deformation of the mild steel plates were studied. Theobald and Nurick [14] studied the responses of tube-core claddings under blast loads by experiments, whose results showed that panel crush distance increases with increasing impulse and decreases with an increasing number of tubes in the panel core. Palanivelu et al. [15] studied crushing and energy absorption performance of different geometrical shapes of small-scale glass polyester composite tubes under quasistatic loading conditions and found that the corresponding energy absorption of the special geometrical shapes was better than that of the standard square and hexagonal geometrical shapes. Tarlochan et al. 
[16] conducted quasistatic compression experimental investigation of composite sandwich cladding structures fabricated from glass fiber, polystyrene foam, and epoxy resin. Zhao et al. [17] proposed a foamed cement-based composite functional material as sacrificial cladding to provide the blast mitigation effect and investigated its response with different ingredient proportions and determined the optimized thickness of foamed cement-based sacrificial cladding.

On the other hand, forming artificial discontinuity functions as a barrier to stress wave transmission is another solution. The artificial discontinuity can be presplit, barrier hole, and a trench. For presplit blasting, Lu et al. [18] studied the vibration isolating mechanism and effect of presplit crack. Lou and Long [19] conducted the field test to study the effect of the presplit in propagation of blasting seismic waves, with the result showing that damping effect of the presplit is related with the distance between the presplit and the point of the seismic wave data collected, and the damping ratio will be in the range of $15 \% \sim 30 \%$. Luo et al. [20] studied the relation between the effect of presplitting crack on damping vibration and the length, depth of presplitting crack, and distance between presplitting crack and blasting source by matlab software. For trench blasting, Fourney et al. [21] investigated the effects of joints and bedding planes on seismic vibration, revealing that as thickness of discontinuity increased, seismic vibration decreased between blasting point and measurement station. Song [22] explained the essence of decreasing vibration methods by energy-origin, energytransfer media, and energy-transfer process. Kattis et al. [23] examined that an open trench was more effective for decreasing vibration than a filled one by research on open and concentrate-filled trenches. Prakash et al. [24] studied effect of trench on vibration level and reported vibration reduction of 16-55\%. Adam and Von Estorff [25] opened trenches near railways and achieved reduction in vibration of up to $80 \%$. Bogunovic and Kecojevic [26] experimentally studied the influence on vibration reduction by different combinations of artificial screen sizes and positions with blasting source and measurement point, making vibration reduce by $30-58 \%$. Ak and Konuk [7] researched impacts of discontinuity frequency parameter derived through geological measurements. The mechanism of blasting seism is complicated with numerous influencing factors. Zhang et al. [27] studied controlling factors of blasting seismic effect by grey correlation theory of grey system and pointed out that the superdeep drilling and pore network parameters are the controlling factors of seismic effect. Uysal et al. [11] conducted a series of field experiments to investigate the effect of barrier holes on blast-induced vibration, and the results revealed that a decrease in PPV of up to $18 \%$ has been detected just behind the barrier holes. Park et al. [28] studied the influence on screening effectiveness by the spacing and diameter of drilling holes and obtained the design details for field application by numerical simulation. Gao et al. [29] studied the vibration-isolation from three dimensions and analyzed the relevant parameters; the results revealed that the reductions are generally more efficient for smaller separation between piles, and the increasing length of piles has a great influence on the reduction of the surface wave field.

Regarding the above studies, it can be concluded that the essence of most common methods for blast-induced vibration reduction is to decrease the initial energy of stress wave or to change stress wave propagation path. Therefore, the method for blast-induced vibration reduction with water jet assistance was put forward by combining with the unique advantages of high-pressure water jet, such as no wear and tear, no spark, energy concentration, and directional cutting. Then, the mechanism of vibration-isolation with water jet assistance was analyzed, and the stress wave energy attenuation models were established based on blasting theory and stress wave theory. Finally, FEM software ANSYS/LSDYNA was used to verify the validity of the theoretical results and qualitatively obtain their influence rules on blast-induced vibration effect.

The differences between the present study and the research performed by other researchers are as follows:

(1) The method for blast-induced vibration reduction with water jet assistance is designed to weaken the effect that stress waves have on the protected objects and is applicable for the occasion in which the protected objects are in the vicinity of blasting source. However, the present technologies aim at weakening the effect that blasting seismic waves have on the protected objects, and, generally comparing with the former method, the work field is further from the protected objects.

(2) This paper focuses on the shock wave energy reduction, while all the above-mentioned researches put emphasis on the seismic wave isolation. In this paper, the model of energy of stress wave was established and demonstrated by FEM software of ANSYS/LS-DYNA.

\section{Experimental Study of Hard Rock Cutting by Water Jet}

Hard rock is a common material and also is an intractable material for the construction of oriented slot and the VIS, since buildings must be constructed on the solid foundation. Therefore, test experiment on cutting hard rock by water jet was conducted to obtain the suitable work parameters. The test system is a set of hard rock cutting experimental system with water jet assistance according to principle of water jet technique. Its schematic diagram is shown in Figure 1. In this experiment, four cone convergent nozzles were adopted. Their convergences are $17^{\circ}$. And their diameters are $2.0 \mathrm{~mm}$, $2.5 \mathrm{~mm}, 3 \mathrm{~mm}$, and $3.5 \mathrm{~mm}$, respectively. The experimental sample $(1 \mathrm{~m} \times 1 \mathrm{~m} \times 0.5 \mathrm{~m}$ in size $)$ is a mixture of sand, cement, and water (mass ratio 1.2:1:2) and is air-dried for 28 days, as shown in Figure 2. The sample has a uniaxial compressive strength of $89 \mathrm{MPa}$, uniaxial tensile strength is $17 \mathrm{Mpa}$, and its Protodikonov hardness coefficient is 9 .

A series of tests have been performed to obtain the relationship between the size of water jet slot and cutting time, pump pressure, and the nozzle diameter. The water 


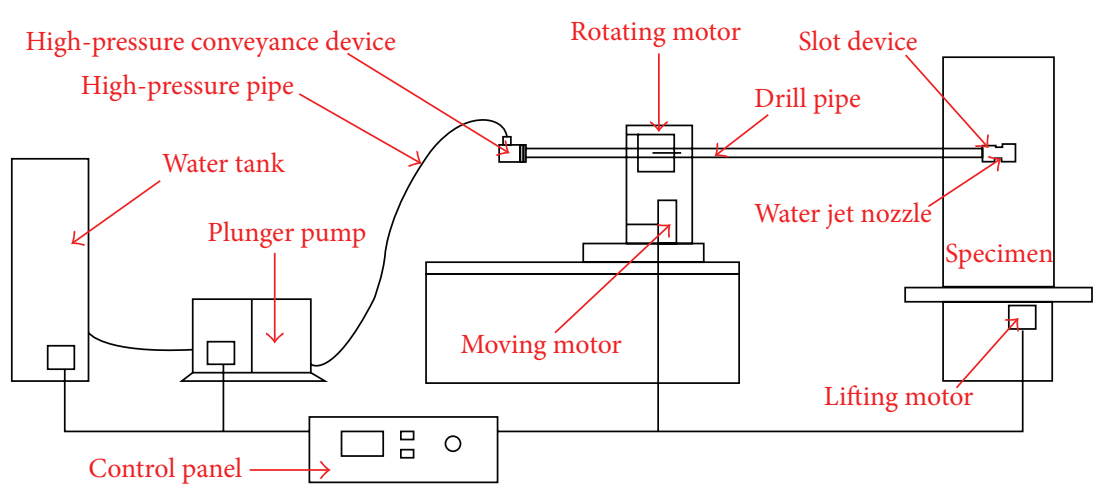

FIGURE 1: Schematic diagram of water jet test system.

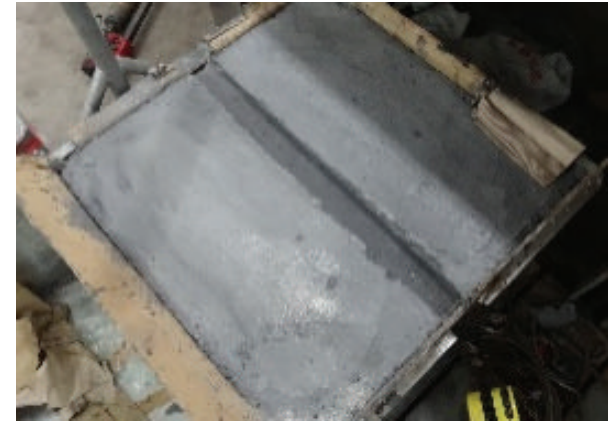

Figure 2: Test specimen.

jet was shown in Figure 3. Depth and width of water jet cutting under each condition are shown in Figures 4 and 5, respectively. As it can be seen from the two figures, with the increase of pump pressure, water jet cutting depth and water jet cutting width increase obviously. Nevertheless, with the increase of pump pressure, growth rate of water jet cutting depth is different. When nozzle diameter is $2.0 \mathrm{~mm}$, water jet cutting depth is $0.1566 \mathrm{~m}$ and $0.4242 \mathrm{~m}$ under the condition of pressure of $10 \mathrm{MPa}$ and $28 \mathrm{MPa}$, and the maximum cutting depth is increased by $170.88 \%$. And when nozzle diameter is $3.5 \mathrm{~mm}$, water jet cutting depth is $0.1123 \mathrm{~m}$ and $0.1722 \mathrm{~m}$, the maximum cutting depth is increased by $53.34 \%$.

On the other hand, with the increase of nozzle diameter, the water jet cutting depth decreases while the water jet cutting width increases. That is because the divergence segment width of water jet increases while the energy of core section of water jet decreases with the increase of nozzle diameter.

\section{Mechanism of Blast-Induced Vibration Reduction Assisted by Water Jet}

3.1. Method for Vibration-Isolation with Assistance of Water Jet Slot. The process of blast-induced vibration reduction with water jet assistance can be divided into the following parts. First, drill boreholes according to design scheme. Second, use water jet to make oriented slots in the surface of the periphery

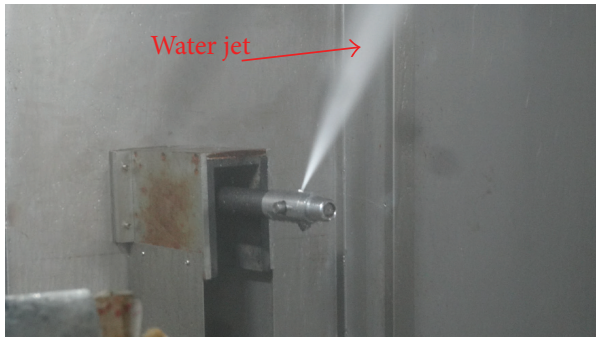

Figure 3: Water jet.

boreholes along the excavation outline. Finally, drill empty holes between the periphery boreholes and the protected objects, and construct the vibration-isolation slot (VIS) with water jet. Its technical sketch was shown in Figure 6.

Previous studies have proved that the slot at the sides of the periphery boreholes can generate stress concentration in the process of explosion and function as a guidance of blasting energy, expanding blast-induced cracks along the excavation outline and reducing stress in other directions. In addition, the VIS can change the propagation path of stress wave, sharply reducing the energy of stress wave which acts on protected object so as to achieve directional fracture and blast-induced vibration reduction.

3.2. Energy Attenuation Models of Stress Wave. Stress wave energy attenuates in the process of propagation and has different attenuation coefficients in the different media. Furthermore, different kinds of stress waves experience attenuation in different degrees [30, 31]. In this paper, major energy attenuation caused by the VIS was focused on and divided into three parts. The first part is the energy dissipating in the form of reflected wave at the first interface between rock and the VIS, which can be expressed by $E_{1}$. The second is energy dissipating during the propagation of stress wave in the VIS and can be expressed by $E_{2}$. The third is the energy dissipating in the form of reflected wave at the second interface between rock and the VIS and can be expressed by $E_{3}$. Assume $E_{t}$ is the total energy reduced by VIS, it can be written as follows:

$$
E_{t}=E_{1}+E_{2}+E_{3} \text {. }
$$




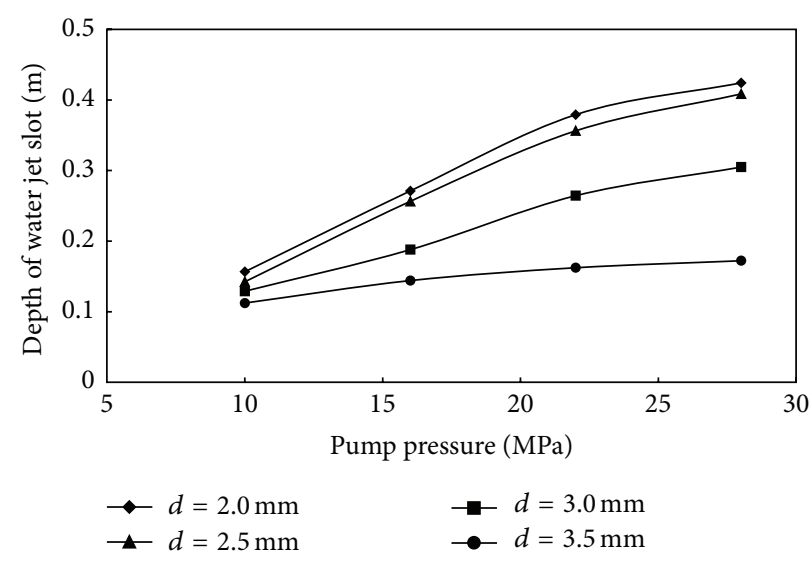

Figure 4: Curve of cutting depth of water jet.

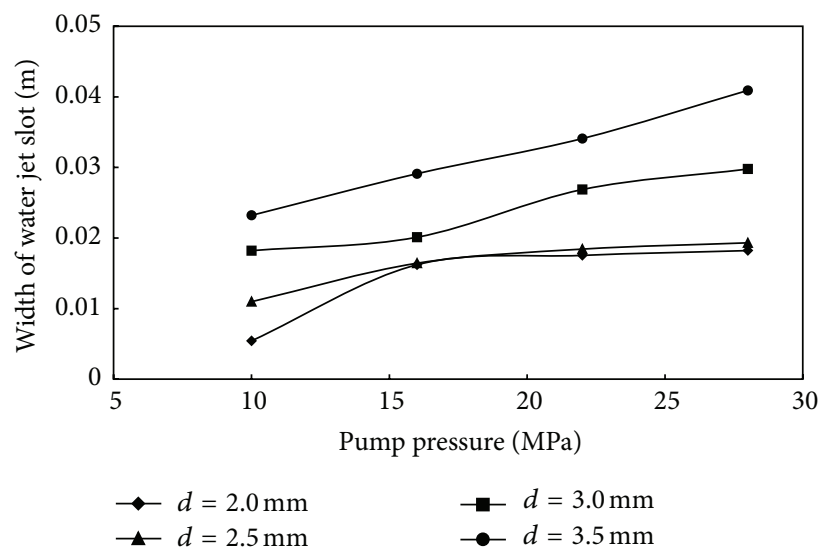

FIGURE 5: Curve of cutting width of water jet.

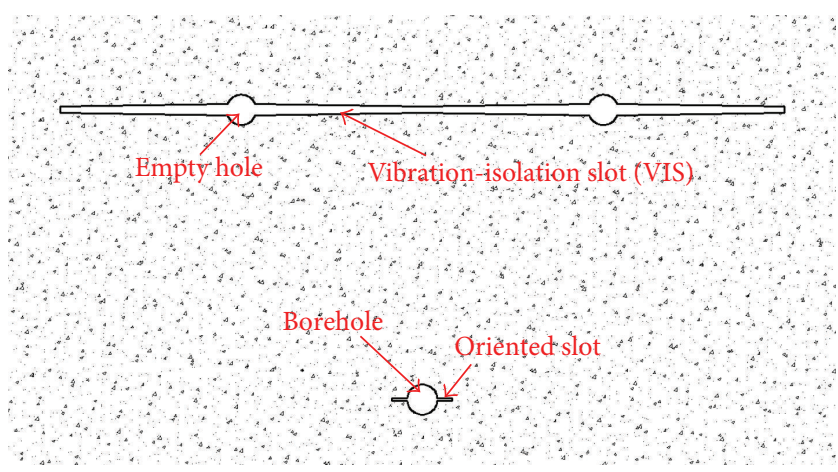

FIGURE 6: Technical sketch of blast-induced vibration reduction with water jet assistance.

According to elasticity theory [32,33], stress wave energy can be calculated by

$$
E=\frac{1}{\rho C} \int_{t_{1}}^{t_{2}} \sigma^{2} d t
$$

where $E$ is the stress wave energy, $\rho$ is density of the medium, $C$ is the velocity of stress wave in the medium, $t_{1}$ is the start time that stress wave starts to act on the medium, $t_{2}$ is the end time that stress wave stops acting on the medium, and $\sigma$ is the stress that acts on the medium.
On the basis of the above analysis of the energy characters of stress wave in the process of propagation, when the VIS is located in the shock wave zone and assuming that the rock was not crushed by explosive shock wave, the relationship between the initial pressure of shock wave produced by explosive and the pressure of shock wave arriving at the VIS can be written as follows:

$$
P_{1}=\sigma_{r}=P_{0}\left(\frac{d}{r_{0}}\right)^{-2-\mu /(1+\mu)},
$$




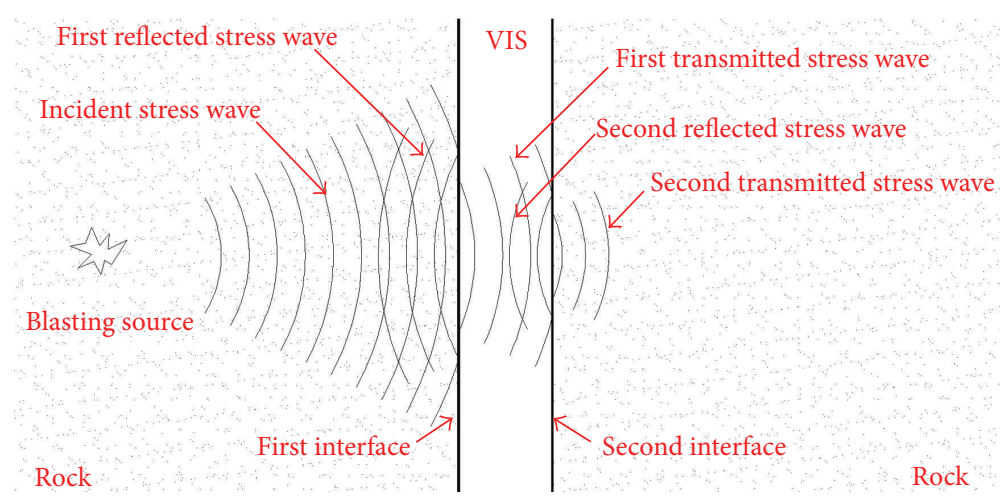

FIGURE 7: Schematic diagram of stress wave propagation.

where $P_{0}$ is the initial pressure of shock wave produced by explosive, $d$ is the distance from explosion source, $\mu$ is Poisson ratio of rock, and $P_{1}$ is the pressure of shock wave arriving at the VIS.

According to blasting theory, if the charge structure of borehole is coupled, the initial pressure of shock wave produced by explosive can be calculated by Formula (4); if the charge structure is uncoupled, the initial pressure of shock wave produced by explosive can be calculated by Formula (5) [34]:

$$
\begin{aligned}
& P_{0}=\frac{2 \rho_{2} C_{2}}{\rho_{e} \nu_{D}+\rho_{2} C_{2}} P_{d}, \\
& P_{0}=\frac{1}{8} \rho_{e} \nu_{D}^{2}\left(\frac{V_{c}}{V_{b}}\right)^{3} n,
\end{aligned}
$$

where $\rho_{2}$ is the density of rock, $\rho_{e}$ is the density of explosive, $v_{D}$ is detonation velocity of explosive, $P_{d}$ is detonation pressure of explosive, and $C_{2}$ is sound velocity of rock. $V_{c}$ is volume of explosive package, $V_{b}$ is volume of explosive compartment, and $n$ is magnification factors and its value is $8 \sim 11$.

In addition, reflection and transmission occur at the interface when stress wave encounters the VIS. Its schematic diagram was shown in Figure 7. And the relationship between incident wave and reflected wave can be written as $[34,35]$

$$
\begin{aligned}
\sigma_{R_{1}} & =\frac{\rho_{2} C_{2}-\rho_{1} C_{1}}{\rho_{2} C_{2}+\rho_{1} C_{1}} \sigma_{I} \\
& =P_{0}\left(\frac{d}{r_{b}}\right)^{-2-\mu /(1+\mu)} \frac{\rho_{2} C_{2}-\rho_{1} C_{1}}{\rho_{2} C_{2}+\rho_{1} C_{1}}, \\
\sigma_{T_{1}} & =\frac{2 \rho_{1} C_{1}}{\rho_{2} C_{2}+\rho_{1} C_{1}} \sigma_{I} \\
& =P_{0}\left(\frac{d}{r_{b}}\right)^{-2-\mu /(1+\mu)} \frac{2 \rho_{1} C_{1}}{\rho_{2} C_{2}+\rho_{1} C_{1}},
\end{aligned}
$$

where $\sigma_{I}$ is incident stress, $\sigma_{R_{1}}$ is reflected wave stress, $\sigma_{T_{1}}$ is transmitted wave stress, $\rho_{1}$ is density of material filling the
VIS, $\rho_{2}$ is density of rock, $C_{1}$ is sound velocity of material filling the VIS, and $C_{2}$ is sound velocity of rock.

Combining with the above formulas, when the VIS is located in the shock wave zone and the charge structure of borehole is coupling charge, then the shock wave energy attenuation caused by the VIS can be calculated by Formula (7). And when the VIS is located in the shock wave zone and the charge structure is coupling charge, then the shock wave energy attenuation caused by the VIS can be calculated by Formula (8):

$$
\begin{aligned}
E_{\mathrm{tsh}} & =\frac{4 \rho_{2} C_{2}\left(\rho_{2} C_{2}-\rho_{1} C_{1}\right)^{2}}{\left(\rho_{e} \nu_{D}+\rho_{2} C_{2}\right)^{2}\left(\rho_{2} C_{2}+\rho_{1} C_{1}\right)^{2}}\left(P_{d}\right)^{2} \\
& \cdot \int_{0}^{t_{4}}\left(\xi_{1}\right)^{2}\left(\frac{d}{r_{b}}\right)^{-4-2 \mu /(1+\mu)} d t \\
& +\frac{16\left(\rho_{2} C_{2}\right)^{2} \rho_{1} C_{1}}{\left(\rho_{e} \nu_{D}+\rho_{2} C_{2}\right)^{2}\left(\rho_{2} C_{2}+\rho_{1} C_{1}\right)^{2}}\left(P_{d}\right)^{2} \\
& \cdot \int_{0}^{t_{5}}\left(\frac{d}{r_{b}}\right)^{-4-2 \mu /(1+\mu)} d t \\
& +\frac{64\left(\rho_{1} C_{1}\right)^{2} \rho_{2} C_{2}}{\left(\rho_{e} \nu_{D}+\rho_{2} C_{2}\right)^{2}\left(\rho_{2} C_{2}+\rho_{1} C_{1}\right)^{4}}\left(P_{d}\right)^{2} \\
\cdot & \int_{0}^{t_{6}}\left(\xi_{1}\right)^{2}\left(\frac{d}{r_{b}}\right)^{-4-2 \mu /(1+\mu)} d t \\
E_{\mathrm{tsh}} & =\frac{\left(\nu_{D}\right)^{4}\left(\rho_{e} n\right)^{2}\left(\rho_{2} C_{2}-\rho_{1} C_{1}\right)^{2}}{64 \rho_{2} C_{2}\left(\rho_{2} C_{2}+\rho_{1} C_{1}\right)^{2}}\left(\frac{V_{c}}{V_{b}}\right)^{6} \\
& \cdot n^{2} \int_{0}^{t_{4}}\left(\xi_{1}\right)^{2}\left(\frac{d}{r_{b}}\right)^{-4-2 \mu /(1+\mu)} d t \\
& +\frac{\left(\nu_{D}\right)^{4}\left(\rho_{e} n\right)^{2} \rho_{1} C_{1}}{16\left(\rho_{2} C_{2}+\rho_{1} C_{1}\right)^{2}}\left(\frac{V_{c}}{V_{b}}\right)^{6} \int_{0}^{t_{5}}\left(\frac{d}{r_{b}}\right)^{-4-2 \mu /(1+\mu)} d t
\end{aligned}
$$




$$
\begin{aligned}
& +\frac{\left(\nu_{D}\right)^{4}\left(\rho_{e} n\right)^{2}\left(\rho_{1} C_{1}\right)^{2}}{4 \rho_{2} C_{2}\left(\rho_{2} C_{2}+\rho_{1} C_{1}\right)^{4}}\left(\frac{V_{c}}{V_{b}}\right)^{3} \\
& \cdot \int_{0}^{t_{6}}\left(\xi_{1}\right)^{2}\left(\frac{d}{r_{b}}\right)^{-4-2 \mu /(1+\mu)} d t,
\end{aligned}
$$

where $E_{\text {tsh }}$ is shock wave energy attenuation caused by the $\mathrm{VIS}, \xi_{1}$ is stress attenuation function of reflected shock wave, $t_{4}$ is the time it takes the first reflected shock wave to act on rock, $t_{5}$ is the time it takes transmitted shock wave to propagate through VIS, and $t_{6}$ is the time it takes second reflected shock wave to act on rock.

In the same way, when the VIS is located in compressive wave zone, the relationship between the initial pressure of shock wave produced by explosive and the pressure of compressive wave arriving at the VIS can be written as follows:

$$
P_{2}=\sigma_{r}=P_{0}\left(\frac{d}{r_{0}}\right)^{-2+\mu /(1-\mu)}
$$

where $P_{2}$ is compressive pressure.

Therefore, when the VIS is located in the compressive wave zone and the charge structure of borehole is coupling charge, the compressive wave energy attenuation by VIS can be calculated by Formula (10). And when the VIS is located in the compressive wave zone and the charge structure of borehole is coupling charge, the compressive wave energy attenuation by VIS can be calculated by Formula (11):

$$
\begin{gathered}
E_{\mathrm{tco}}=\frac{4 \rho_{2} C_{2}\left(\rho_{2} C_{2}-\rho_{1} C_{1}\right)^{2}}{\left(\rho_{e} \nu_{D}+\rho_{2} C_{2}\right)^{2}\left(\rho_{2} C_{2}+\rho_{1} C_{1}\right)^{2}}\left(P_{d}\right)^{2} \\
\cdot \int_{0}^{t_{7}}\left(\xi_{2}\right)^{2}\left(\frac{d}{r_{b}}\right)^{-4+2 \mu /(1-\mu)} d t \\
+\frac{16 \rho_{1} C_{1}\left(\rho_{2} C_{2}\right)^{2}}{\left(\rho_{e} \nu_{D}+\rho_{2} C_{2}\right)^{2}\left(\rho_{2} C_{2}+\rho_{1} C_{1}\right)^{2}}\left(P_{d}\right)^{2} \\
\cdot \int_{0}^{t_{8}}\left(\frac{d}{r_{b}}\right)^{-4+2 \mu /(1-\mu)} d t \\
+\frac{64 \rho_{2} C_{2}\left(\rho_{1} C_{1}\right)^{2}}{\left(\rho_{e} \nu_{D}+\rho_{2} C_{2}\right)^{2}\left(\rho_{2} C_{2}+\rho_{1} C_{1}\right)^{4}}\left(P_{d}\right)^{2} \\
\cdot \int_{0}^{t_{9}}\left(\xi_{2}\right)^{2}\left(\frac{d}{r_{b}}\right)^{-4+2 \mu /(1-\mu)} d t \\
\cdot \int_{0}^{t_{7}}\left(\xi_{1}\right)^{2}\left(\frac{d}{r_{b}}\right)^{-4+2 \mu /(1-\mu)} d t \\
=\frac{\left(\rho_{e} n\right)^{2}\left(\nu_{D}\right)^{4}\left(\rho_{2} C_{2}-\rho_{1} C_{1}\right)^{2}}{64 \rho_{2} C_{2}\left(\rho_{2} C_{2}+\rho_{1} C_{1}\right)^{2}}\left(\frac{V_{c}}{V_{b}}\right)^{6}
\end{gathered}
$$

$$
\begin{aligned}
& +\frac{\left(\rho_{e} n\right)^{2}\left(\nu_{D}\right)^{4} \rho_{1} C_{1}}{16\left(\rho_{2} C_{2}+\rho_{1} C_{1}\right)^{2}}\left(\frac{V_{c}}{V_{b}}\right)^{6} \\
& \cdot \int_{0}^{t_{8}}\left(\frac{d}{r_{b}}\right)^{-4+2 \mu /(1-\mu)} d t \\
& +\frac{\left(\rho_{e} n\right)^{2}\left(\nu_{D}\right)^{4}\left(\rho_{1} C_{1}\right)^{2}}{4 \rho_{2} C_{2}\left(\rho_{2} C_{2}+\rho_{1} C_{1}\right)^{2}}\left(\frac{V_{c}}{V_{b}}\right)^{6} \\
& \cdot \int_{0}^{t_{9}}\left(\xi_{1}\right)^{2}\left(\frac{d}{r_{b}}\right)^{-4+2 \mu /(1-\mu)} d t,
\end{aligned}
$$

where $E_{\text {tco }}$ is compressive wave energy attenuation caused by the VIS, $\xi_{2}$ is stress attenuation function of reflected compressive wave. $t_{7}$ is the time it takes the first reflected compressive wave to act on rock, $t_{8}$ is the time it takes transmitted compression wave to propagate through VIS, $t_{9}$ is the time it takes the second reflected compressive wave to act on rock.

By analyzing Formula (7), Formula (8), Formula (10), and Formula (11), some conclusions can be drawn as bellow:

(1) In Formula (7), except for $d$, other parameters can be seen as constants and they are greater than zero identically. On the contrary, $-2+\mu /(1-\mu)$ is less than zero. Therefore, with the increase of $d$, the shock wave energy attenuation caused by the VIS decreases. Similarly, Formula (8), Formula (10), and Formula (11) share the same trend. In other words, the smaller the distance between borehole and VIS, the better the blast-induced reduction effect achieved.

(2) $\left(16\left(\rho_{2} C_{2}\right)^{2} \rho_{1} C_{1} /\left(\rho_{e} \nu_{D}+\rho_{2} C_{2}\right)^{2}\left(\rho_{2} C_{2}+\right.\right.$ $\left.\left.\rho_{1} C_{1}\right)^{2}\right)\left(P_{d}\right)^{2} \int_{0}^{t_{5}}\left(d / r_{b}\right)^{-4-2 \mu /(1+\mu)} d t$ stands for the energy dissipating during the propagation of stress wave in the VIS, and $t_{5}$ is the time it takes transmitted shock wave to propagate through VIS. Therefore, with the increase of the width of VIS, the energy dissipating during this process increases, and the better blast-induced vibration reduction effect will be achieved.

(3) The smaller the wave impedance of material filling VIS is, the bigger the stress wave energy attenuation of VIS is. In other words, the material with low wave impedance is suitable for the material filling VIS to achieve the good blast-induce vibration effect.

\section{Numerical Simulations}

In this section, FEM software (ANSYS/LS-DYNA) was used for numerical simulation studies on blasting-induced vibration reduction effect influenced by the distance between VIS and borehole and the material filling VIS, which aims at verifying the validity of the theoretical results and qualitatively obtaining their influence rules on blast-induced vibration effect. 
TABle 1: Case defined.

\begin{tabular}{lccc}
\hline Case number & $D(\mathrm{~m})$ & Material filling the VIS & Material filling the oriented slot \\
\hline 1 & 0.1 & Air & Air \\
2 & 0.2 & Air & Air \\
3 & 0.3 & Air & Air \\
4 & 0.4 & Air & Air \\
5 & 0.5 & Air & Air \\
6 & 0.1 & Water & Air \\
7 & 0.2 & Water & Air \\
8 & 0.3 & Water & Air \\
9 & 0.4 & Water & Air \\
10 & 0.5 & Air & \\
\hline
\end{tabular}

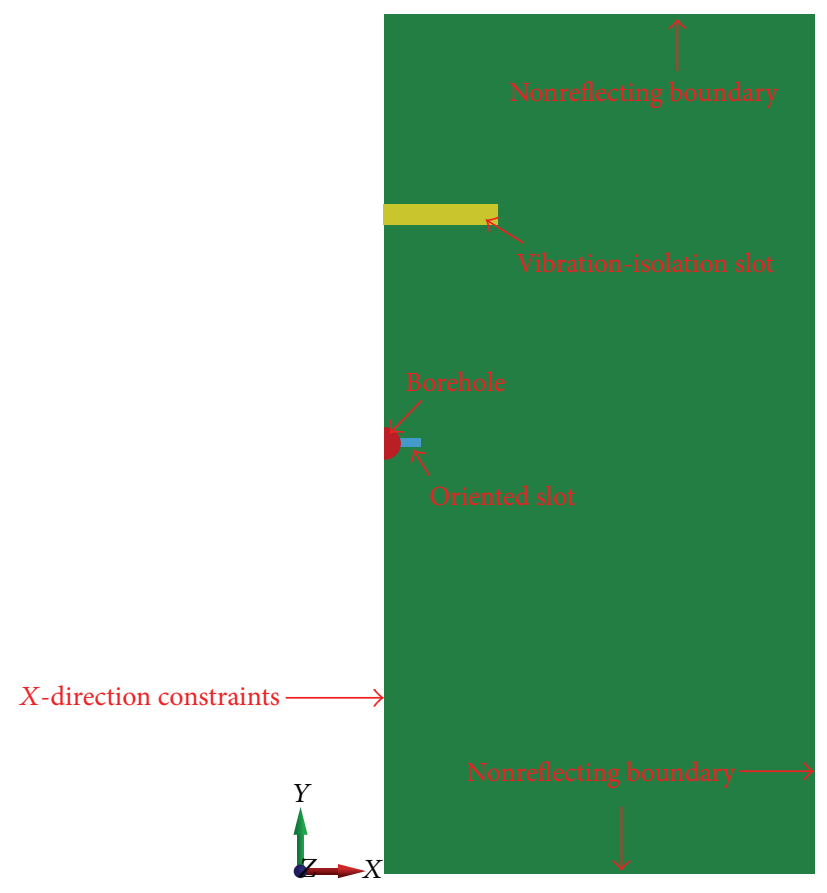

Figure 8: Numerical simulation model of Case 4.

4.1. Simulation Model and Simulated Cases. The blast-induced vibration reduction effect of area near blasting source is numerically simulated in ten different cases to compare the contribution of the VIS, in which we define $D$ as the distance between VIS and borehole, and the ten cases are shown in Table 1.

Due to the symmetry of the simulated object, a half model with symmetrical constraints in $X$ direction was applied. And in each case, rock is $0.75 \mathrm{~m} \times 1.5 \mathrm{~m} \times 0.03 \mathrm{~m}$ in size, borehole is $0.05 \mathrm{~m}$ in diameter, oriented slot is $0.04 \mathrm{~m} \times 0.03 \mathrm{~m} \times 0.03 \mathrm{~m}$ in size, and the VIS is $0.2 \mathrm{~m} \times 0.05 \mathrm{~m} \times 0.03 \mathrm{~m}$ in size. The simulation model of Case 4 is shown as in Figure 8.

To avoid the large deformation problem of explosive during the blasting process, fluid-solid coupling algorithm was adopted, in which ALE algorithm is used for explosive and material filling oriented slot, Lagrange algorithm for rock, and material filling VIS. At the same time, meshes of explosive and air filling oriented slot were joined with common nodes,
TABLE 2: Parameters of rock.

\begin{tabular}{cccccc}
\hline$\rho_{2}\left(\mathrm{~kg} / \mathrm{m}^{3}\right)$ & $E(\mathrm{MPa})$ & $P_{R}$ & $Y_{\mathrm{SIG}}(\mathrm{MPa})$ & $E_{\mathrm{TAN}}(\mathrm{MPa})$ & $F_{S}$ \\
\hline $2.55 \times 10^{3}$ & $2.25 \times 10^{4}$ & 0.22 & 3.24 & $4.25 \times 10^{3}$ & 0.06 \\
\hline
\end{tabular}

and the meshes of rock and the material filling VIS share the same nodes. Then, the fluid-solid coupling was defined by the keyword of CONSTRAINED_LAGRANGE_IN_SOLID [36].

On the other hand, according to the characteristics of blasting process, the time step of the simulation is 0.67 , and the computation time is $0.002 \mathrm{~s}$.

\subsection{Material Model}

4.2.1. Material Model for Rock. According to experiment test on physical property of rock, material type 3 of LS-DYNA ( ${ }^{*}$ MAT_PLASTIC_KINEMATIC) is suitable, and Table 2 
TABLE 3: Parameters of explosive and its EOS equation.

\begin{tabular}{lccccccc}
\hline$\rho_{e}\left(\mathrm{~kg} / \mathrm{m}^{3}\right)$ & $\nu_{D}(\mathrm{~m} / \mathrm{s})$ & $P_{\text {cut }}(\mathrm{MPa})$ & $A(\mathrm{MPa})$ & $B(\mathrm{MPa})$ & $R_{1}$ & $R_{2}$ & $\omega$ \\
\hline $1.93 \times 10^{3}$ & $9.93 \times 10^{3}$ & $3.37 \times 10^{4}$ & $3.71 \times 10^{5}$ & $7.43 \times 10^{3}$ & 4.15 & 0.95 & 0.30 \\
\hline
\end{tabular}

TABLE 4: Parameters of air and its EOS equation.

\begin{tabular}{cccccccccc}
\hline$\rho_{a}\left(\mathrm{~kg} / \mathrm{m}^{3}\right)$ & $C_{0}$ & $C_{1}$ & $C_{2}$ & $C_{3}$ & $C_{4}$ & $C_{5}$ & $C_{6}$ & $E_{a 0}$ & $V_{a 0}$ \\
\hline $1.25 \times 10^{3}$ & $-1 \times 10^{-6}$ & 0.00 & 0.00 & 0.00 & 0.40 & 0.40 & 0.00 & $2.5 \times 10^{-6}$ & 1.0 \\
\hline
\end{tabular}

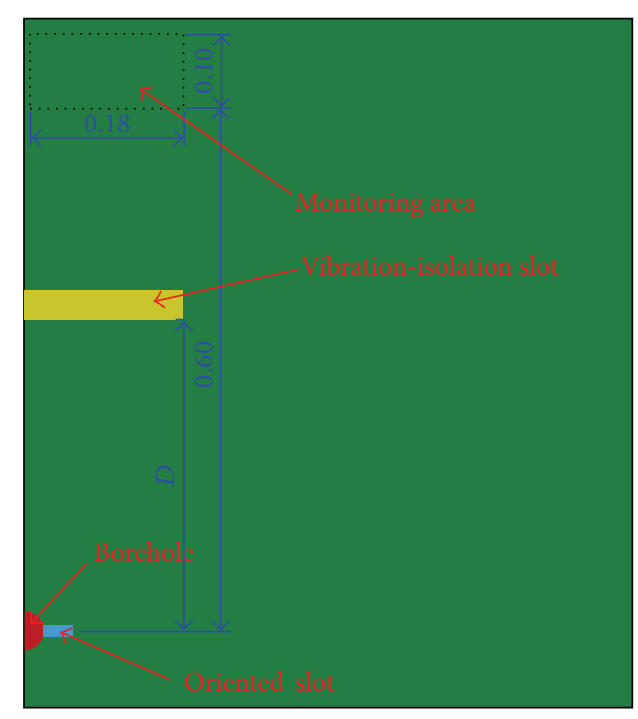

FIGURE 9: Layout of monitoring area.

gives its parameters, where $\rho_{2}$ is density of rock, $E$ is Young modulus, $P_{R}$ is Poisson ratio of rock, $Y_{\text {SIG }}$ is Yield stress of rock, $E_{\text {TAN }}$ is Tangent modulus, and $F_{S}$ is Failure strain for eroding elements [37].

4.2.2. Material Model for Explosive. Explosive was modeled by the material type 8 of LS-DYNA ( ${ }^{*} \mathrm{MAT}_{-} \mathrm{HIGH}_{-}$ EXPLOSIVE_BURN) [37], and the Jones-Wilkens-Lee (JWL) equation of state was used for the pressure applied by the detonation product gases. It can be calculated by

$$
\begin{aligned}
P_{e}= & A\left(1-\frac{\omega}{R_{1} V_{e}}\right) e^{-R_{1} V_{e}}+B\left(1-\frac{\omega}{R_{2} V_{e}}\right) e^{-R_{2} V_{e}} \\
& +\frac{\omega E_{e}}{V_{e}}
\end{aligned}
$$

where $P_{e}$ is pressure applied by the detonation product gases, $\rho_{e}$ is density of explosive, $\nu_{D}$ is detonation velocity of explosive, $P_{\text {cut }}$ is Chapman-Jouget pressure, $\omega, A, B, R_{1}$, and $R_{2}$ are user defined input parameters, $V_{e}$ is relative volume, and $E_{e}$ is internal energy per initial volume, as shown in Table 3.
4.2.3. Material Model for Air. Air was modeled by material type 9 of LS-DYNA ( ${ }^{*}$ MAT_NULL) with LINEAR_POLYNOMIAL equation, the pressure $P_{a}$ can be calculated by [37]

$$
\begin{aligned}
P_{a}= & C_{0}+C_{1} \mu+C_{2} \mu^{2}+C_{3} \mu^{3} \\
& +\left(C_{4}+C_{5} \mu+C_{6} \mu^{2}\right) E_{a}, \quad \mu=\frac{1}{V_{a}}-1,
\end{aligned}
$$

where $C_{0}, C_{1}, C_{2}, C_{3}, C_{4}, C_{5}$, and $C_{6}$ are user defined constants, $\rho_{a}$ is density of air, $V_{a}$ is the relative volume, $V_{a 0}$ is the initial relative volume of air, $E_{a}$ is internal energy, and $E_{a 0}$ is the initial internal energy of air, as shown in Table 4.

4.2.4. Material Model for Water. The water was modeled by the material type 9 of LS-DYNA ( ${ }^{*}$ MAT_NULL) with Gruneisen equation, the pressure $P_{w}$ can be calculated by [37]

$$
\begin{aligned}
& P_{w} \\
& =\frac{\rho_{0} C^{2} \mu\left[1+\left(1-\gamma_{0} / 2\right) \mu-(a / 2) \mu^{2}\right]}{\left[1-\left(S_{1}-1\right) \mu-S_{2}\left(\mu^{2} /(\mu+1)\right)-S_{3}\left(\mu^{3} /(\mu+1)^{2}\right)\right]^{2}} \\
& \quad+\left(\gamma_{0}+a \mu\right) E_{w}, \quad \mu=\frac{\rho}{\rho_{0}}-1,
\end{aligned}
$$




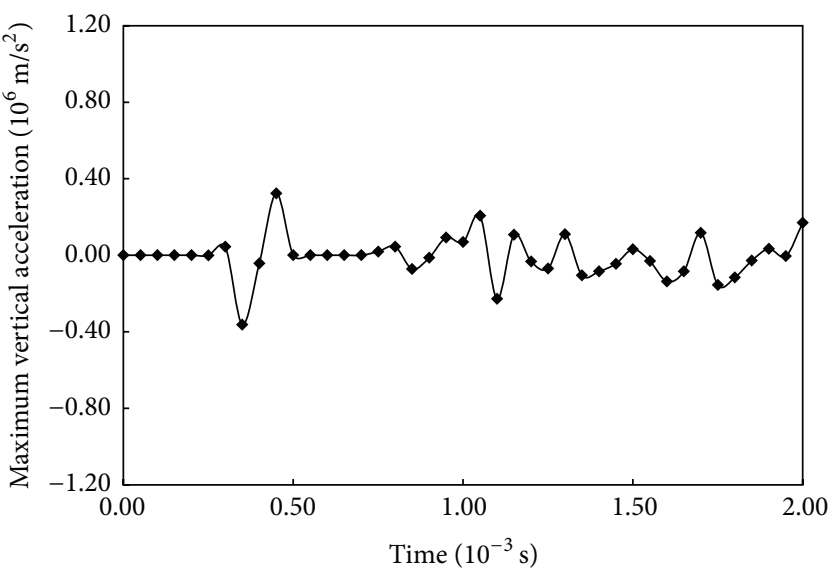

(a) Maximum vertical acceleration

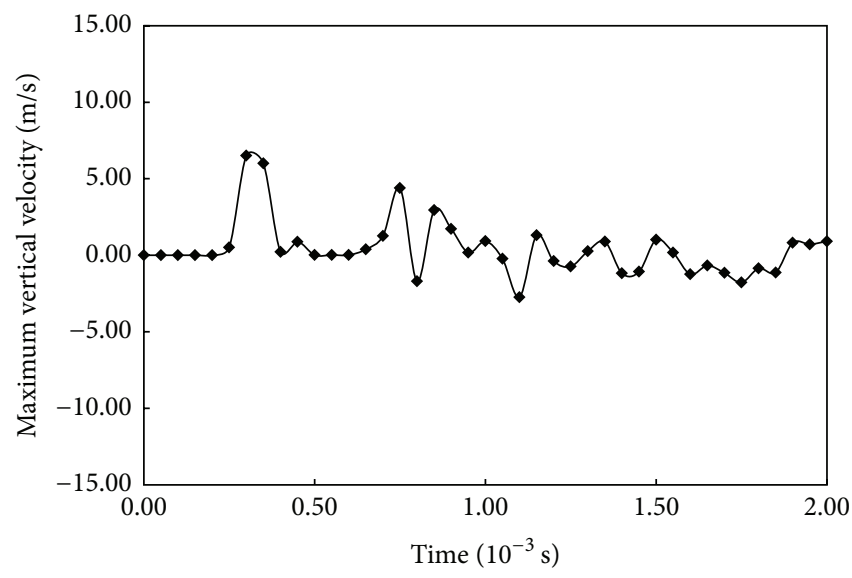

(b) Maximum vertical velocity

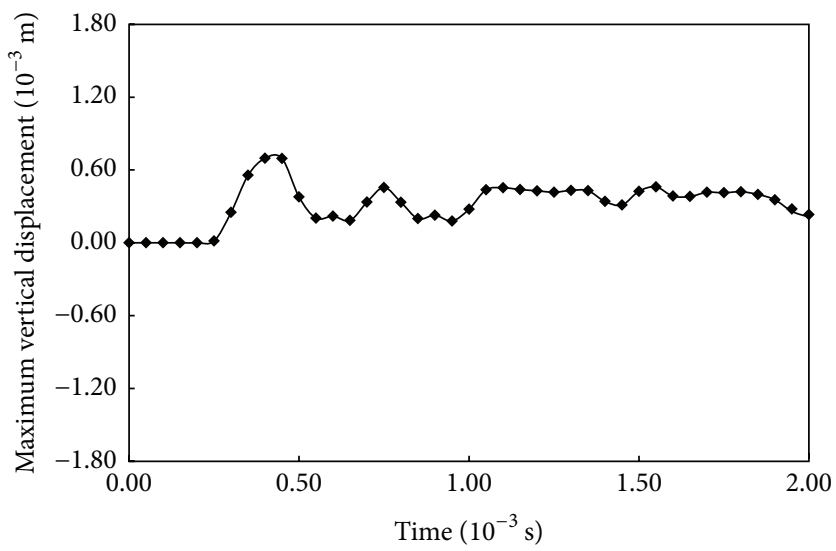

(c) Maximum vertical displacement

FIGURE 10: Blast-induced vibration parameters of Case 1.

where $E_{w}$ is internal energy per initial volume, $\rho_{w}$ is density of water, $C$ is intercept of $u_{s}-u_{p}$ curve, $S_{1}, S_{2}$, and $S_{3}$ are coefficients of the slope of the $u_{s}-u_{p}$ curve, $\gamma_{0}$ is the Gruneisen gamma, $a$ is the first-order volume correction to $\gamma_{0}$, constants $S_{1}, S_{2}$, and $S_{3}, \gamma_{0}$, and $a$ are all input parameters, and $E_{w 0}$ is the initial internal energy of water, as shown in Table 5.

\subsection{Numerical Results and Discussions}

4.3.1. Influence of Distance between VIS and Borehole. The effect of varying the distance between borehole and VIS of the blast-induced vibration was investigated by varying the distance between borehole and VIS from $0.1 \mathrm{~m}$ to $0.5 \mathrm{~m}$ at $0.1 \mathrm{~m}$ intervals. Meanwhile, a rectangular region $(0.1 \mathrm{~m} \times 0.18 \mathrm{~m})$ behind the VIS was set as monitoring area for blast-induced vibration condition of stress wave under each condition, as shown in Figure 9. The distance between monitored area and explosion source remained
TABLE 5: Parameters of water and its EOS equation.

\begin{tabular}{cccccc}
\hline$\rho_{w}\left(\mathrm{~kg} / \mathrm{m}^{3}\right)$ & $C$ & $S_{1}$ & $S_{2}$ & $S_{3}$ & $E_{w 0}$ \\
\hline $1.02 \times 10^{3}$ & 1.65 & 1.92 & $-0.96 \times 10^{-1}$ & 0.00 & 0.00 \\
\hline
\end{tabular}

$0.6 \mathrm{~m}$, and we define $D$ as the distance between VIS and borehole. Then, the maximum vertical acceleration, maximum vertical velocity, and maximum vertical displacement in the monitored area under each condition were drawn in Figures 10-14.

Figures 10 14 show the blast-induced vibration parameters under each condition. Compared with the curves in Figures 10 14, with the increase of distance between the VIS and borehole, the vibration parameters almost show a linear increase. When the distance between the VIS and explosion source is $0.1 \mathrm{~m}$, the maximum acceleration, maximum velocity, and maximum displacement are $3.23 \times$ $10^{5} \mathrm{~m} / \mathrm{s}^{2}, 6.52 \mathrm{~m} / \mathrm{s}$, and $6.97 \times 10^{-4} \mathrm{~m}$, respectively. When the 


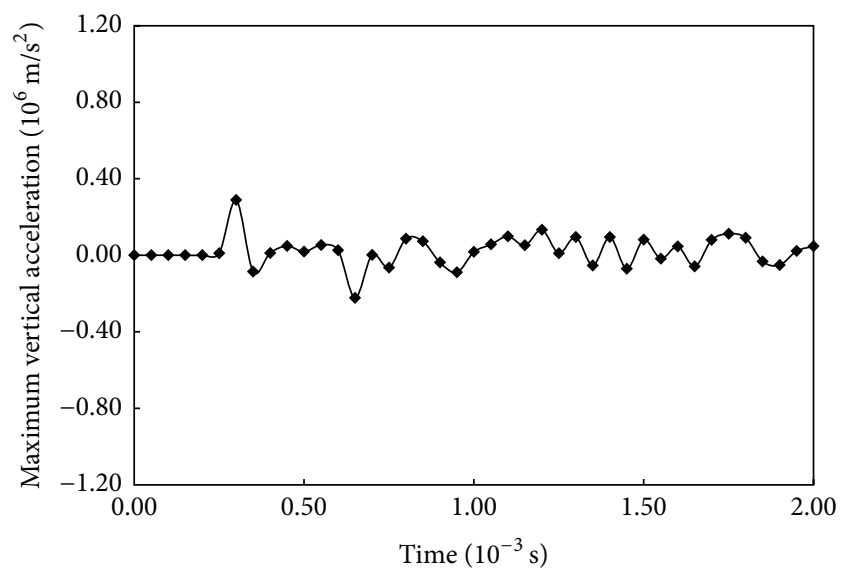

(a) Maximum vertical acceleration

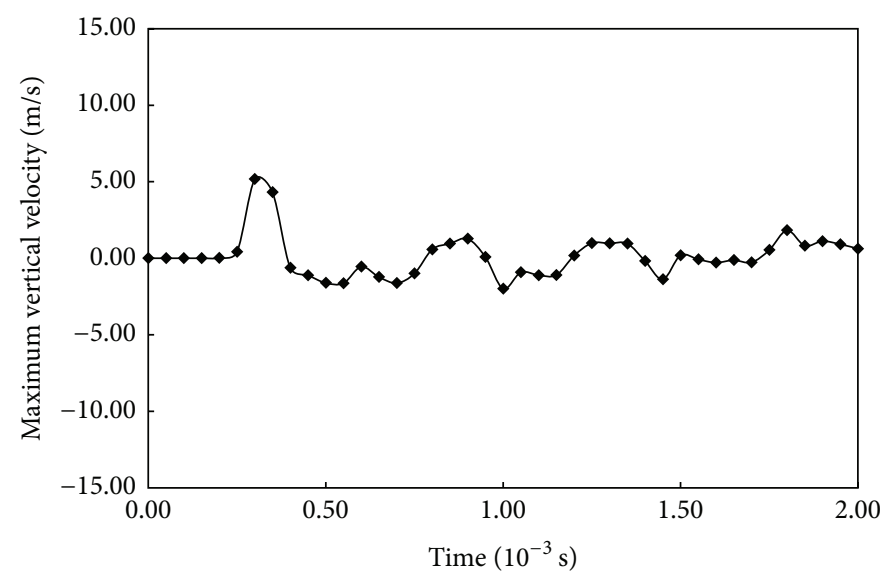

(b) Maximum vertical velocity

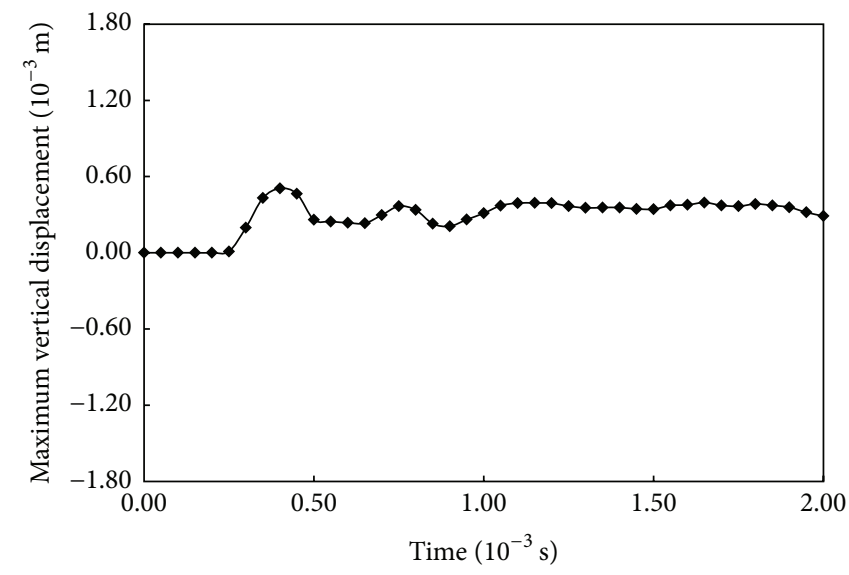

(c) Maximum vertical displacement

FIGURE 11: Blast-induced vibration parameters of Case 2.

distance between VIS and borehole is $0.5 \mathrm{~m}$, the maximum acceleration, maximum velocity, and maximum displacement are $9.92 \times 10^{5} \mathrm{~m} / \mathrm{s}^{2}, 1.02 \times 10 \mathrm{~m} / \mathrm{s}$, and $1.66 \times 10^{-3} \mathrm{~m}$, respectively, and the parameters increase by $207.12 \%, 56.44 \%$, and $138.16 \%$, respectively. Particularly, compared with the vibration parameters under the condition of distance $0.1 \mathrm{~m}$ and vibration parameters under the condition of distance $0.2 \mathrm{~m}$, the vibration parameters decrease with the increase of distance. When the distance between the VIS and borehole is $0.2 \mathrm{~m}$, the maximum acceleration, maximum velocity, and maximum displacement decrease by $10.84 \%, 20.71 \%$, and $27.4 \%$, respectively. It is because the distance between VIS and borehole is too small, which leads to the rock being crushed by shock wave and sharply reducing the energy of shock wave after it goes through the VIS, as shown in Figure 15. Therefore, it can be concluded that when the distance between the protected objects and blasting source remains certain, the smaller the distance is, the more efficient the blast-induced effect is. This is also confirmed by Section 3.

4.3.2. Influence of Material Filling the VIS. Attenuation ratio of vibration parameters can be calculated by [34]

$$
\eta=\frac{c-d}{c} * 100 \%
$$

where $\eta$ is attenuation ratio, $c$ is maximum blast-induced vibration parameter in front of VIS, $d$ is maximum blastinduced vibration parameter behind VIS. The attenuation ratio of vibration parameters are shown in Tables 6 and 7, and the relationship between distance and attenuation ratio under each condition is shown in Figure 16. 


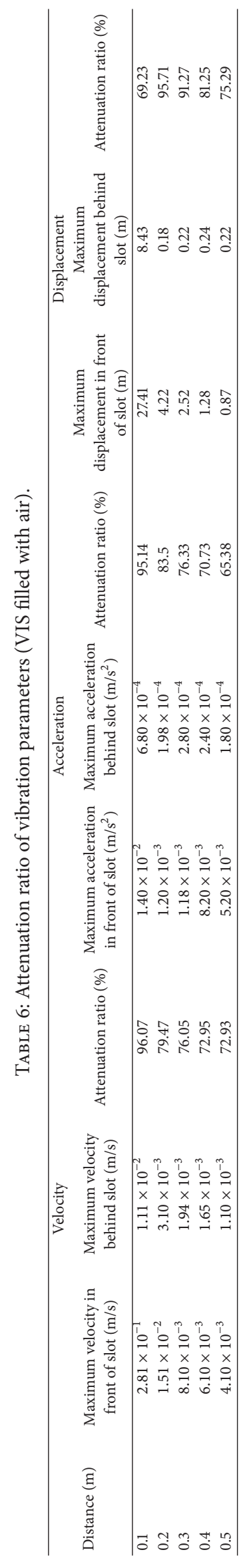




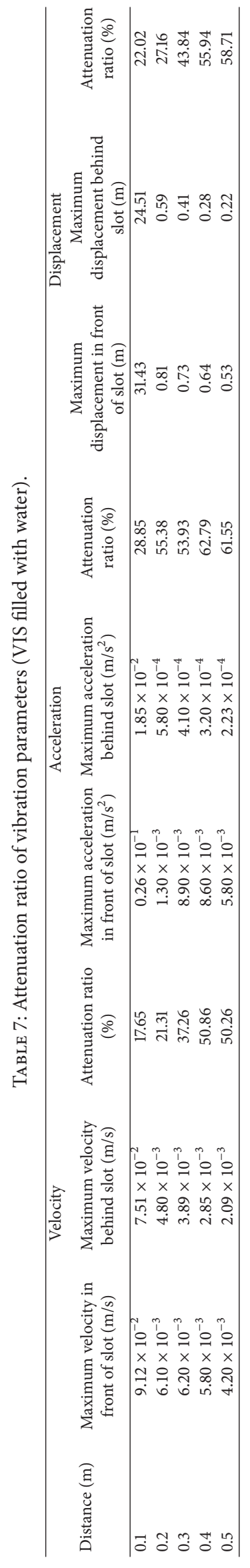




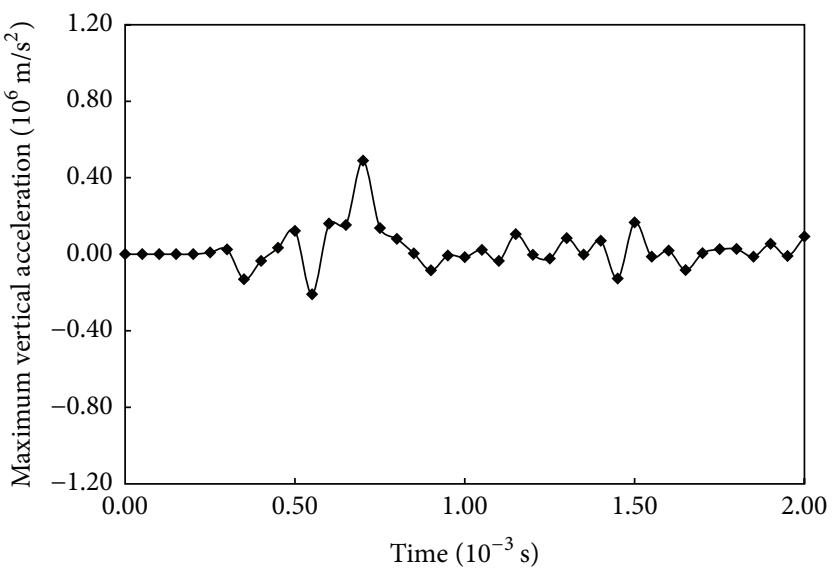

(a) Maximum vertical acceleration

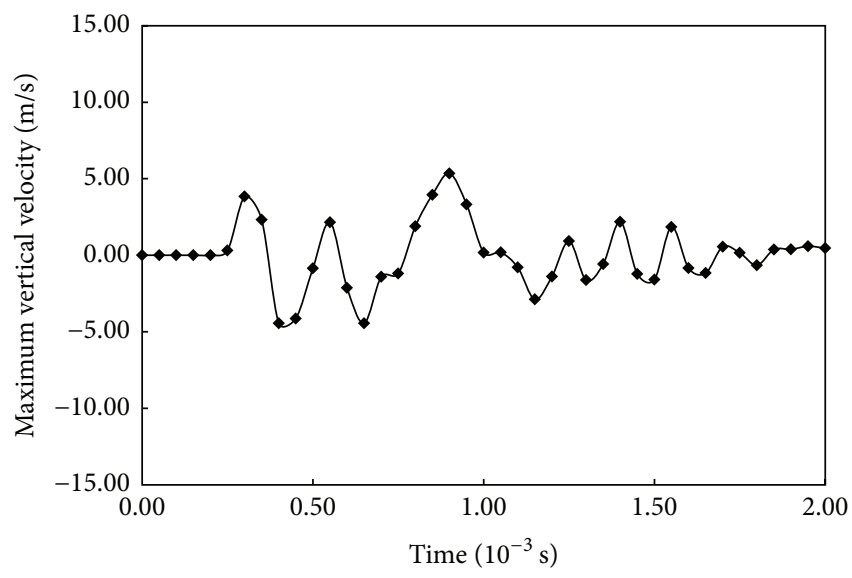

(b) Maximum vertical velocity

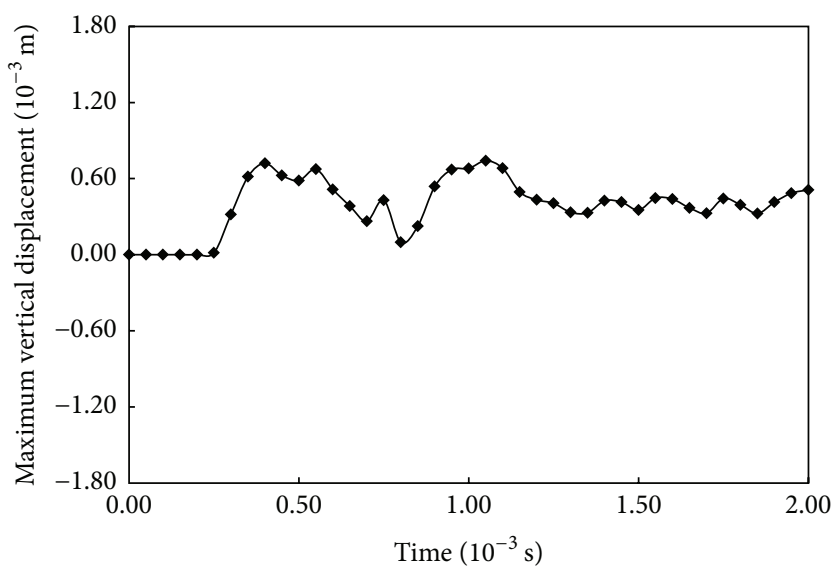

(c) Maximum vertical displacement

FIGURE 12: Blast-induced vibration parameters of Case 3.

Figure 16 shows the attenuation ratio of VIS under each condition. For the VIS filled with air, its attenuation ratio decreases with increase of distance between VIS and borehole. Particularly, with the increase of the distance between VIS and borehole, attenuation ratio of displacement shows a tendency to increase at first and then decrease. And for the VIS with water, its attenuation ratio increases with the increase of distance between VIS and borehole. These trends above agree with theoretical analysis roughly.

On the other hand, compared with Figures 16(a) and $16(\mathrm{~b})$, attenuation ratio of vibration parameters caused by VIS filled with air is greater than that caused by VIS filled with water. When the distance from VIS to explosion source is $0.2 \mathrm{~m}$, attenuation ratios of acceleration, velocity, and displacement caused by VIS filled with air are increased by $20.88 \%, 68.76 \%$, and $71.63 \%$, respectively, compared with those caused by VIS filled with water. The reason for that is because air is different from water in compressibility. Figures 17 and 18 present the evolution law of shock wave when it propagated through the VIS. As it can be seen when shock wave propagate through VIS filled with air, reflection and transmission occur at the interface, shock wave energy sharply reduces, and shock wave form is also changed. However, when shock wave propagates through VIS filled with water, shock wave form is barely changed.

In addition, sharply reducing the distance between VIS and borehole is not a good choice to achieve good directional blasting effect and blast-induced vibration reduction effect. This is because if the distance from VIS to explosion source is too short, the rock will be crushed by shock wave when the reflected wave stress exceeds the dynamic tensile strength of rock and because the process can further affect the directional blasting effect by oriented slot, just as shown in Figure 17. Therefore, it is necessary to set the suitable distance and choose material filling VIS so as to achieve good directional blasting effect and blast-induced vibration reduction effect. 


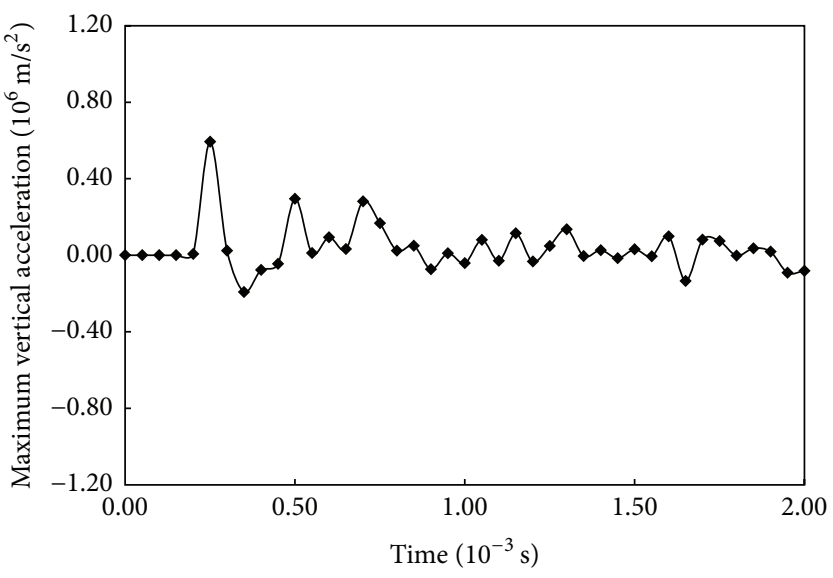

(a) Maximum vertical acceleration

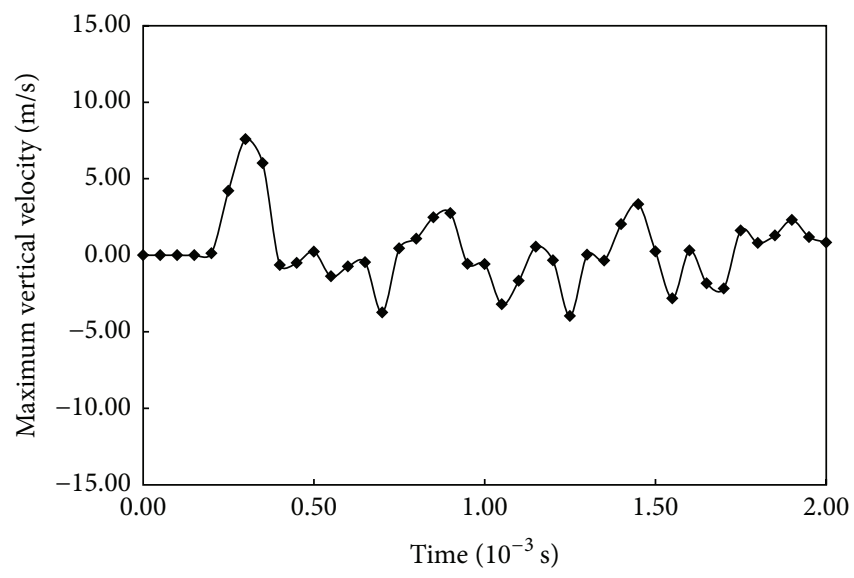

(b) Maximum vertical velocity

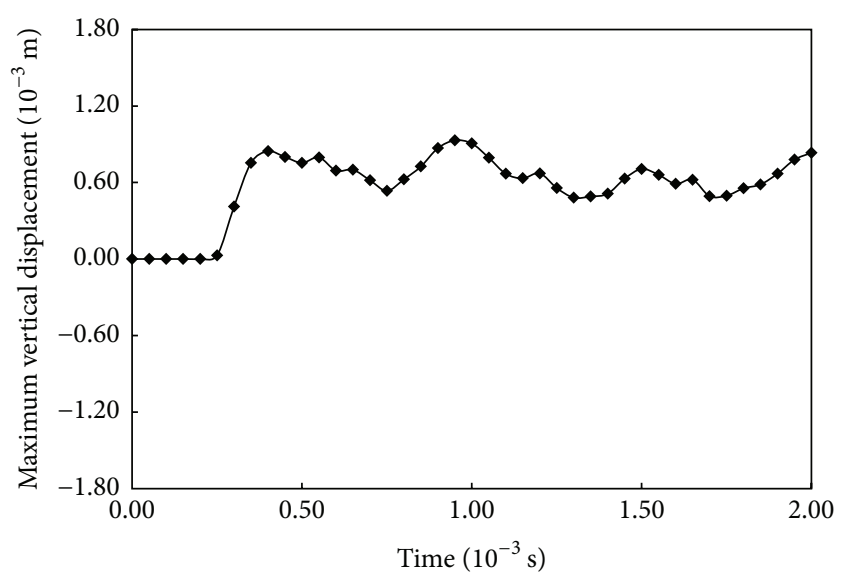

(c) Maximum vertical displacement

FIGURE 13: Blast-induced vibration parameters of Case 4.

\section{Conclusions}

(1) Blast-induced vibration reduction method with water jet assistance was put forward. Hard rock experiment was conducted to obtain the suitable work parameter. The mechanism of vibration-isolation by water jet was analyzed, and the stress wave energy attenuation model by VIS near blasting source was established according to stress wave theory and blast theory.

(2) Influence law on shock wave attenuation by VIS was simulated by ANSYS/LS-DYNA. The results indicate that, for the VIS filled with water, attenuation ratio of vibration parameters increases with the increase of distance between VIS and borehole. For the VIS filled with air, with the decrease of distance between VIS and borehole, attenuation ratio of vertical acceleration and vertical velocity increase, and attenuation ratio of displacement, however, shows a tendency of increase at first and then decrease. In addition, under the same condition, attenuation ratio of blastinduced vibration parameters for VIS filled with air is greater than the attenuation ratio of blastinduced vibration parameter for VIS filled with water. The critical value for distance between borehole and VIS filled with air can achieve good directional blasting effect and blast-induced vibration reduction effect.

In this study, the verification of shock wave energy attenuation model and the studies of influence rules on blastinduced vibration effect were only performed numerically. Therefore, in the future, the laboratory experimental and the field experimental study to verify the results will be performed.

\section{Competing Interests}

The authors declare that there is no conflict of interests regarding the publication of this paper. 


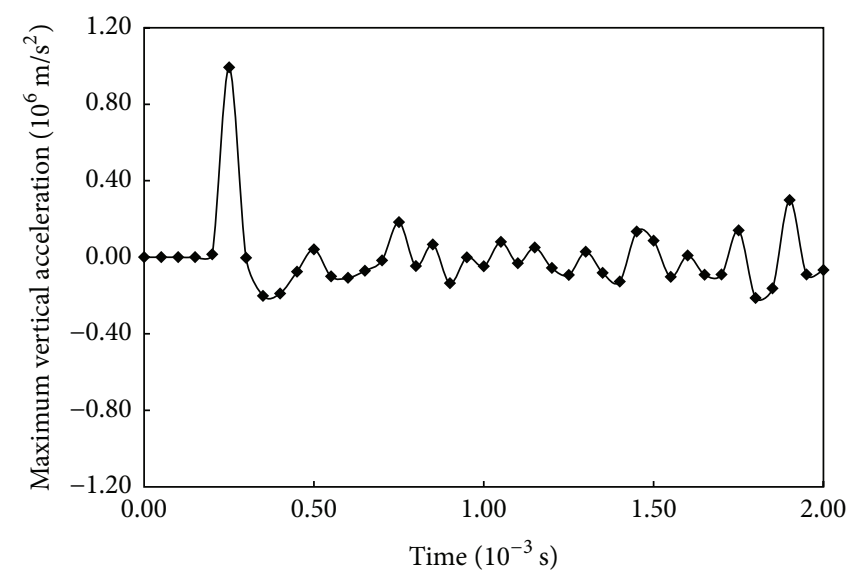

(a) Maximum vertical acceleration

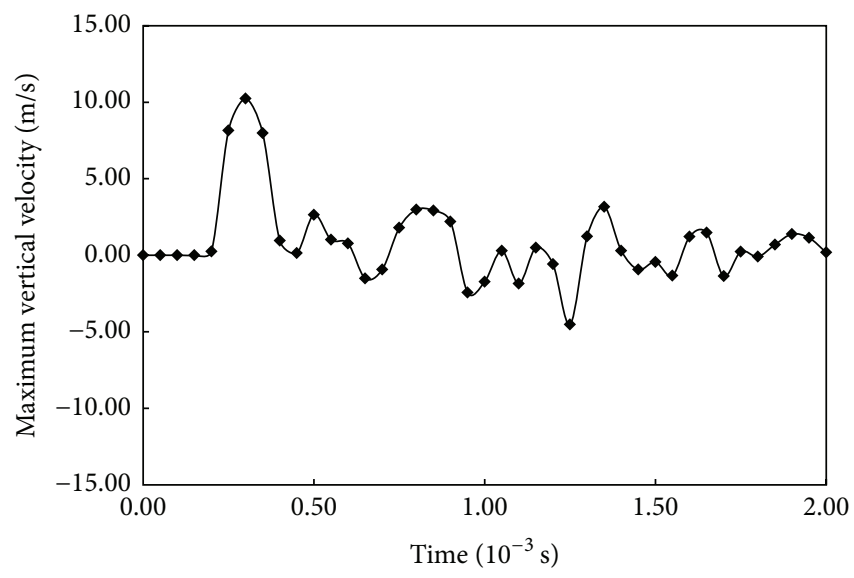

(b) Maximum vertical velocity

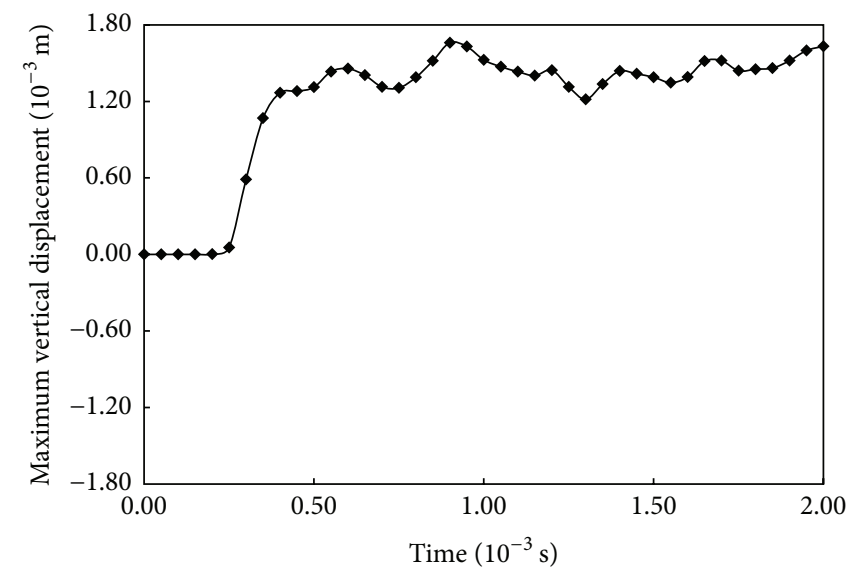

(c) Maximum vertical displacement

FIgURE 14: Blast-induced vibration parameters of Case 5.

LS-DYNA user input

Time $=119.93$

Contours of pressure

Min $=-0.00133656$, at elem $\# 67891$

Max $=0.00535616$, at elem\# 72055

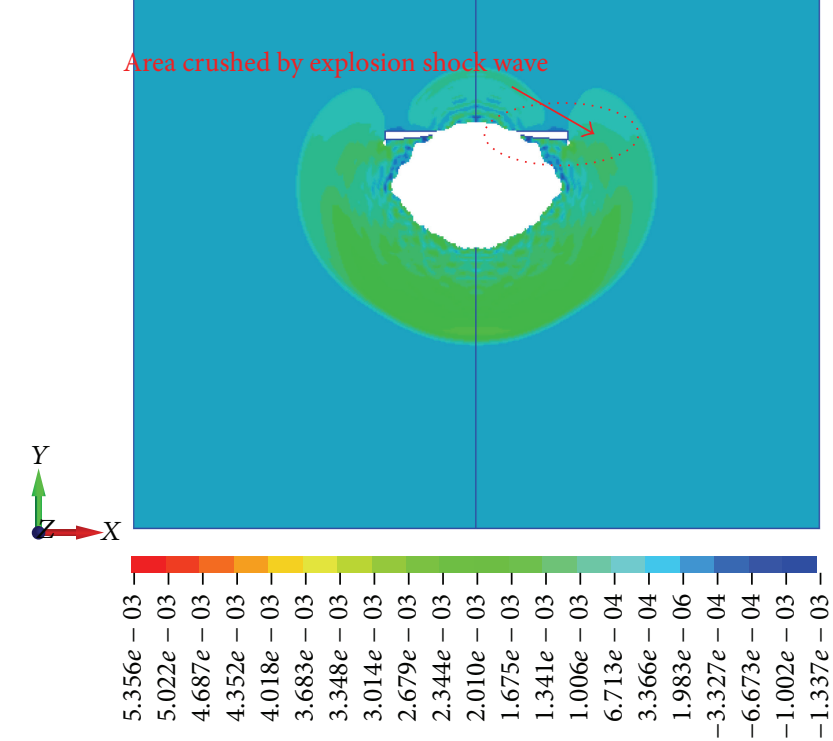

Fringe levels

(a) $t=120 \mu \mathrm{s}$

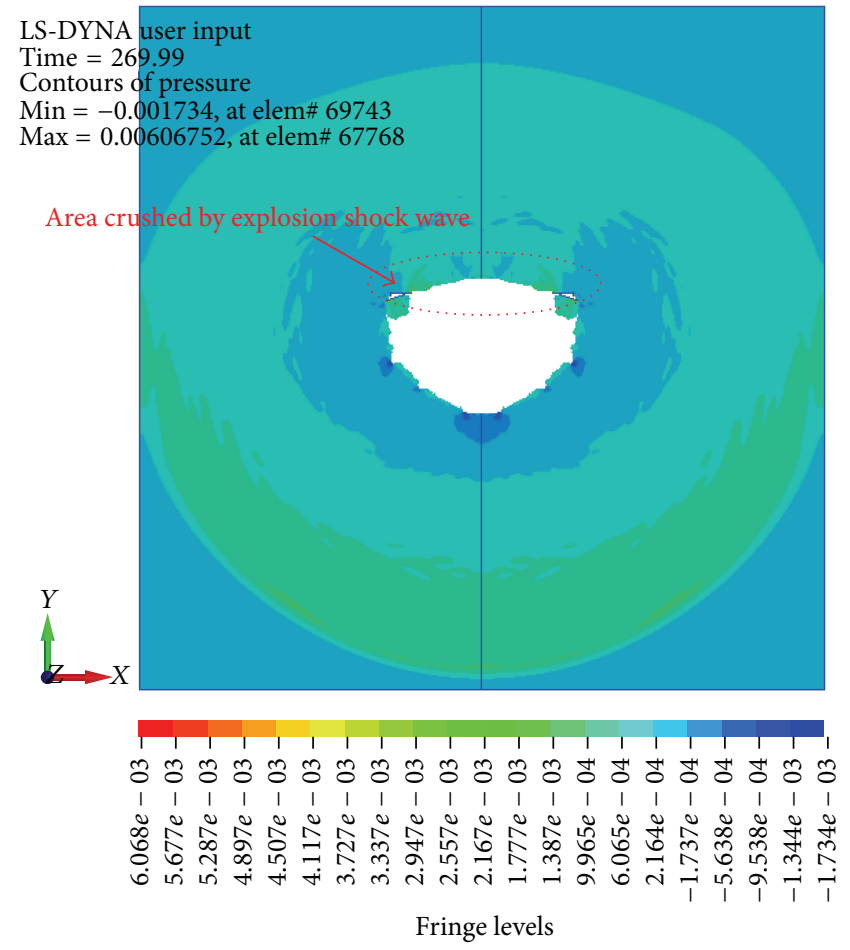

(b) $t=270 \mu \mathrm{s}$

FIgURE 15: Diagram of crushed zone formation $(D=0.1 \mathrm{~m})$. 


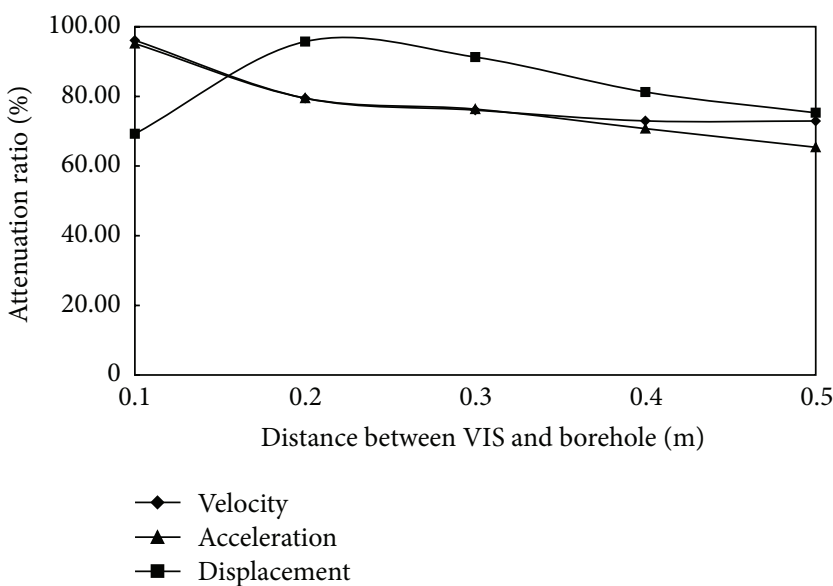

(a) VIS filled with air

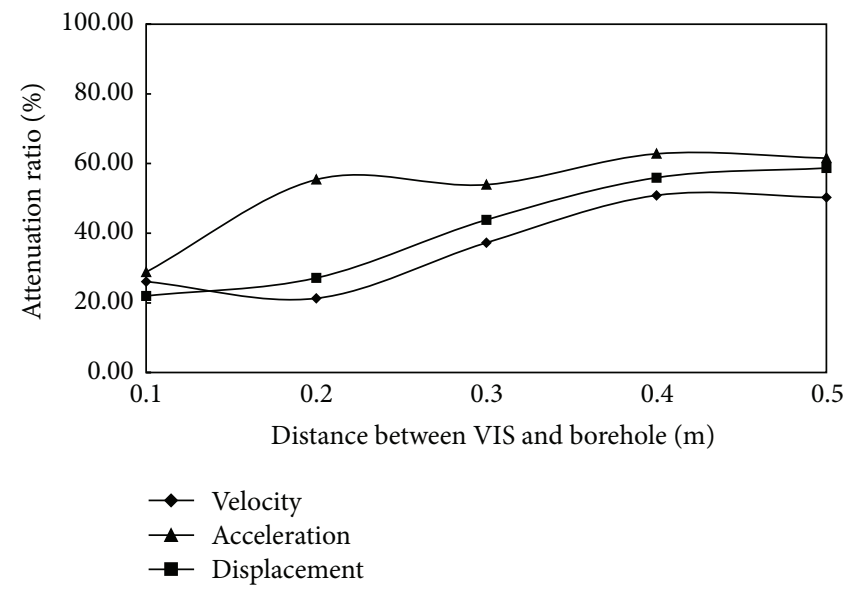

(b) VIS filled with water

FIgURE 16: Curve of attenuation ratio under each condition.

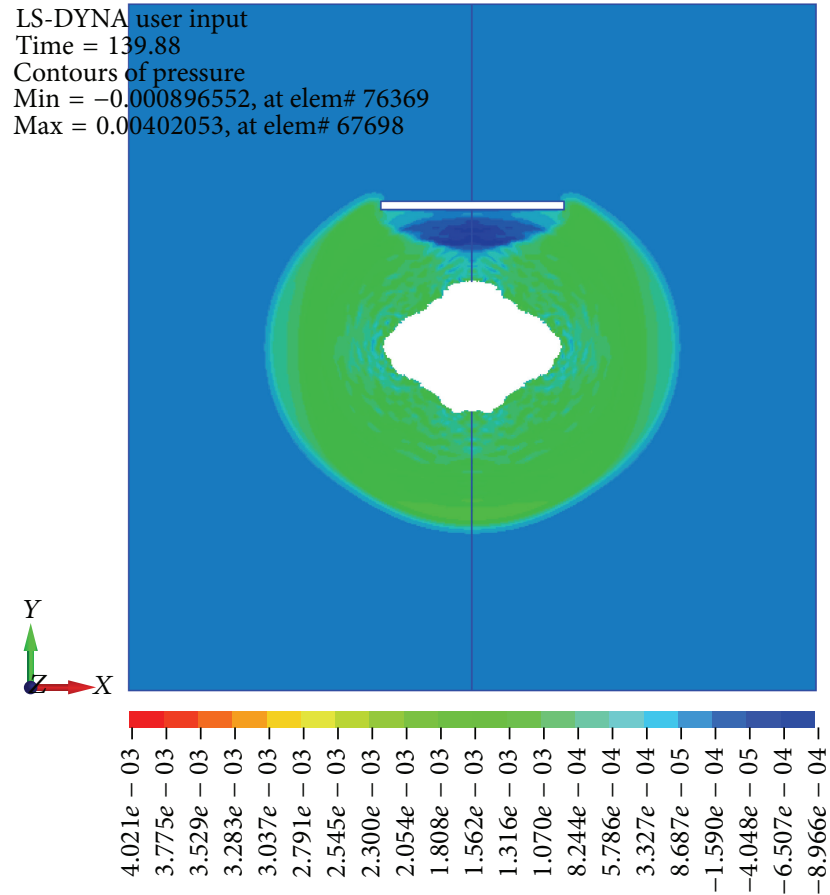

Fringe levels

(a) $t=120 \mu \mathrm{s}$

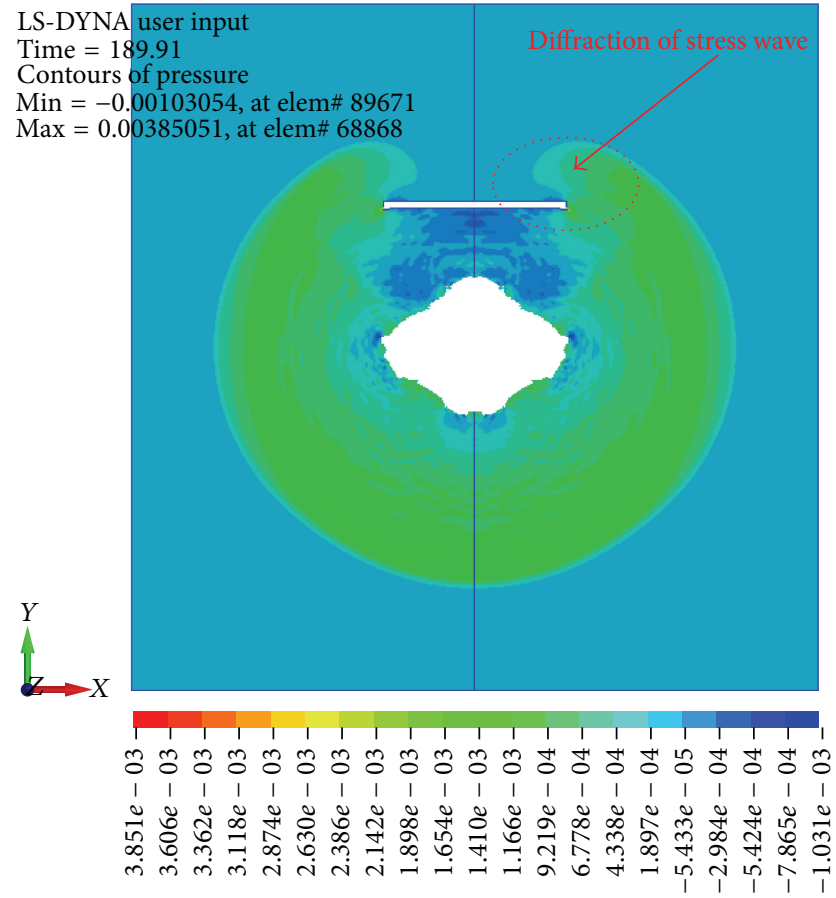

Fringe levels

(b) $t=190 \mu \mathrm{s}$

FIGURE 17: Evolution of shock wave through VIS filled with air $(D=0.3 \mathrm{~m})$.

\section{Acknowledgments}

This paper is jointly supported by National Key Basic Research Program of China (no. 2014CB239203), Program for New Century Excellent Talents in University
(NCET-12-0424), and the Open Projects of State Key Laboratory of Coal Mine Disaster Dynamics and Control (Chongqing University 2011DA105287-FW201205). The authors would like to thank the State Key Laboratory of Coal Mine Disaster Dynamics and Control (Chongqing 


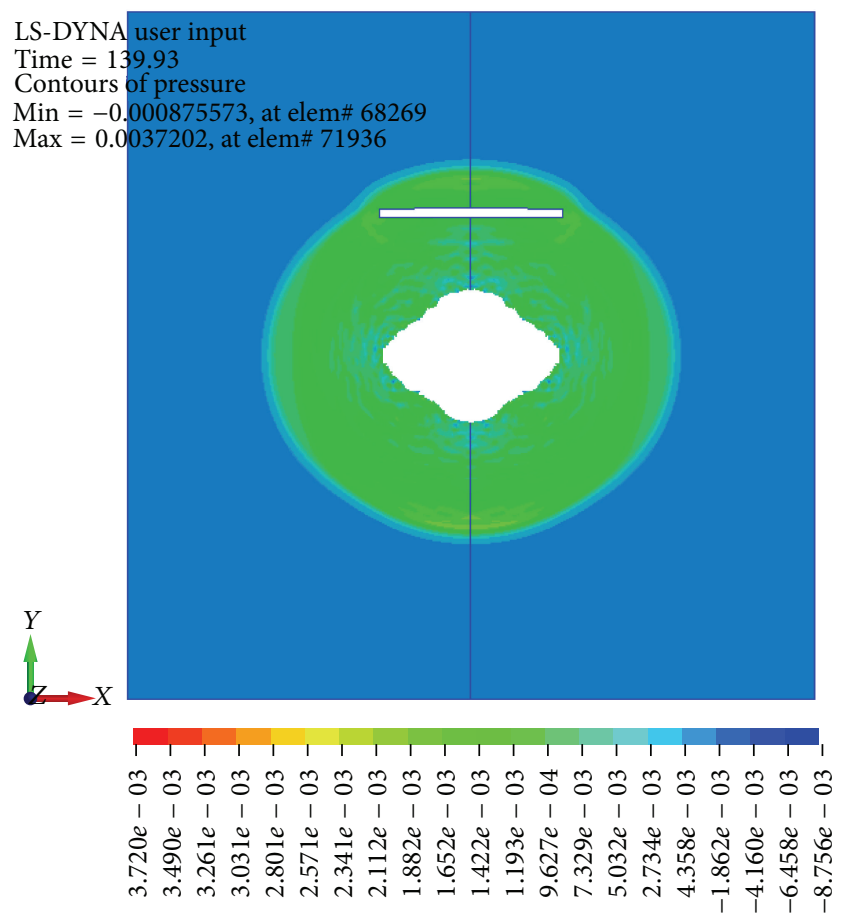

Fringe levels

(a) $t=120 \mu \mathrm{s}$

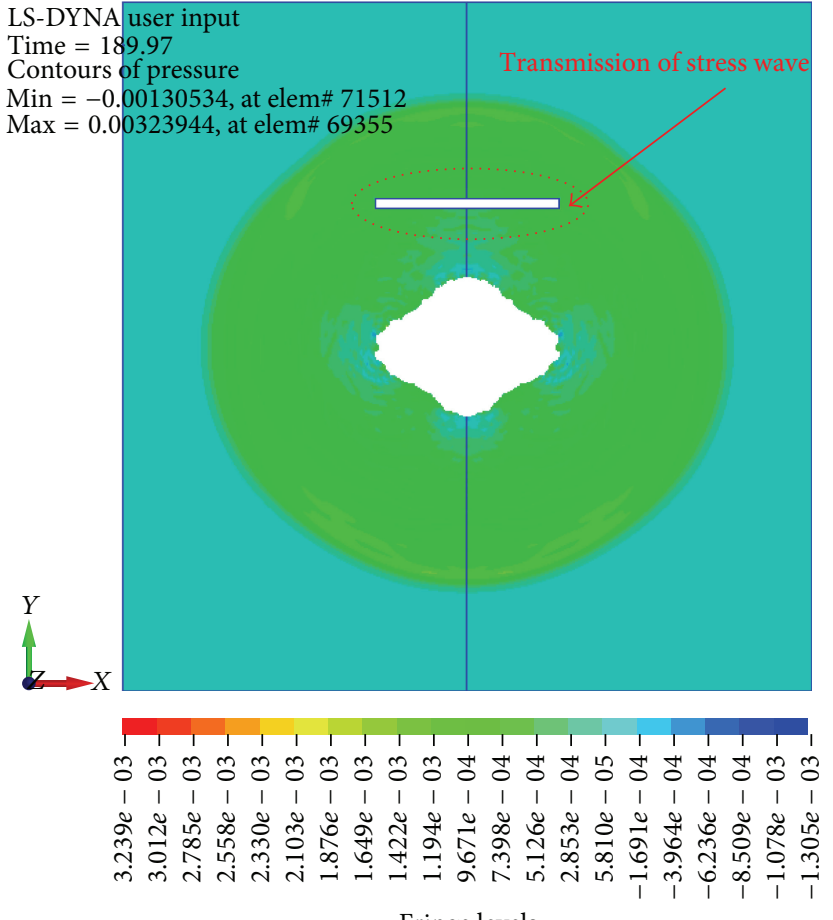

Fringe levels

(b) $t=190 \mu \mathrm{s}$

FIGURE 18: Evolution of shock wave through VIS filled with water $(D=0.3 \mathrm{~m})$.

University) and Hubei Key Laboratory of Waterjet Theory and New Technology (Wuhan University).

\section{References}

[1] T. N. Singh and V. Singh, "An intelligent approach to prediction and control ground vibration in mines," Geotechnical and Geological Engineering, vol. 23, no. 3, pp. 249-262, 2005.

[2] S. Jones, Transportation and Construction-Induced Vibration Guidance Manual, California Department of Transport, Noise, Vibration, and Hazardous Waste Management Office, Sacramento, Calif, USA, 2004.

[3] W. Cao, X. Li, M. Tao, and Z. Zhou, "Vibrations induced by high initial stress release during underground excavations," Tunnelling and Underground Space Technology, vol. 53, pp. 7895, 2016.

[4] H. Ak, M. Iphar, M. Yavuz, and A. Konuk, "Evaluation of ground vibration effect of blasting operations in a magnesite mine," Soil Dynamics and Earthquake Engineering, vol. 29, no. 4, pp. 669676, 2009.

[5] C. Kuzu and E. Guclu, "The problem of human response to blast induced vibrations in tunnel construction and mitigation of vibration effects using cautious blasting in half-face blasting rounds," Tunnelling and Underground Space Technology, vol. 24, no. 1, pp. 53-61, 2009.

[6] M. Monjezi, M. Ghafurikalajahi, and A. Bahrami, "Prediction of blast-induced ground vibration using artificial neural networks," Tunnelling and Underground Space Technology, vol. 26, no. 1, pp. 46-50, 2011.

[7] H. Ak and A. Konuk, "The effect of discontinuity frequency on ground vibrations produced from bench blasting: a case study,"
Soil Dynamics and Earthquake Engineering, vol. 28, no. 9, pp. 686-694, 2008.

[8] A. P. Rustan, "Micro-sequential contour blasting-how does it influence the surrounding rock mass?" Engineering Geology, vol. 49, no. 3-4, pp. 303-313, 1998.

[9] Z. Wang, C. Fang, Y. Chen, and W. Cheng, "A comparative study of delay time identification by vibration energy analysis in millisecond blasting," International Journal of Rock Mechanics and Mining Sciences, vol. 60, pp. 389-400, 2013.

[10] M. Monjezi, M. Ahmadi, M. Sheikhan, and A. Bahrami, "Predicting blast induced ground vibration using various types of neural networks," Soil Dynamics and Earthquake Engineering, vol. 30, no. 11, pp. 33-63, 2010.

[11] O. Uysal, K. Erarslan, M. Akif Cebi, and H. Akcakoca, "Effect of barrier holes on blast induced vibration," International Journal of Rock Mechanics and Mining Sciences, vol. 45, no. 5, pp. 712719, 2008.

[12] X. Z. Shi and S. R. Chen, "Delay time optimization in blasting operations for mitigating the vibration-effects on final pit walls' stability," Soil Dynamics and Earthquake Engineering, vol. 31, no. 8, pp. 1154-1158, 2011.

[13] S. Guruprasad and A. Mukherjee, "Layered sacrificial claddings under blast loading. Part I-analytical studies," International Journal of Impact Engineering, vol. 24, no. 9, pp. 957-973, 2000.

[14] M. D. Theobald and G. N. Nurick, "Experimental and numerical analysis of tube-core claddings under blast loads," International Journal of Impact Engineering, vol. 37, no. 3, pp. 333-348, 2010.

[15] S. Palanivelu, W. V. Paepegem, J. Degrieck et al., "Crushing and energy absorption performance of different geometrical shapes of small-scale glass/polyester composite tubes under 
quasi-static loading conditions," Composite Structures, vol. 93, no. 2, pp. 992-1007, 2011.

[16] F. Tarlochan, S. Ramesh, and S. Harpreet, "Advanced composite sandwich structure design for energy absorption applications: blast protection and crashworthiness," Composites Part B: Engineering, vol. 43, no. 5, pp. 2198-2208, 2012.

[17] H. Zhao, H. Yu, Y. Yuan, and H. Zhu, "Blast mitigation effect of the foamed cement-base sacrificial cladding for tunnel structures," Construction and Building Materials, vol. 94, no. 6891, pp. 710-718, 2015.

[18] W. B. Lu, S. H. Lai, and Z. H. Dong, "Analysis of vibration isolating effect of pre-slitting cracking in rock excavation by blasting," Explosion and Shock Waves, vol. 17, pp. 193-198, 1997.

[19] J. W. Lou and Y. Long, "Study on the effect of the pre-splitting propagation of the blasting seismic waves," Blasting, vol. 22, pp. $1-5,2005$.

[20] Y. Luo, Y. Y. Liu, and C. P. Yi, "Numerical simulation analysis of the effect of pre-splitting crack parameters on damping vibration," Blasting, vol. 24, pp. 25-27, 2007.

[21] W. L. Fourney, R. D. Dick, D. F. Fordyce, and T. A. Weaver, "Effects of open gaps on particle velocity measurements," Rock Mechanics and Rock Engineering, vol. 30, no. 2, pp. 95-111, 1997.

[22] G. M. Song, "Approach to effect of blast vibration and methods to decrease vibration," The World Mining Letters, vol. 15, pp. 2629, 1999.

[23] S. E. Kattis, D. Polyzos, and D. E. Beskos, "Modelling of pile wave barriers by effective trenches and their screening effectiveness," Soil Dynamics and Earthquake Engineering, vol. 46, pp. 713-728, 1999.

[24] A. J. Prakash, P. Palroy, and D. D. Misra, "Analysis of blast vibration characteristics across a trench and a pre-split plane," Fragblast, vol. 8, no. 1, pp. 51-60, 2004.

[25] M. Adam and O. Von Estorff, "Reduction of train-induced building vibrations by using open and filled trenches," Computers and Structures, vol. 83, no. 1, pp. 11-24, 2005.

[26] D. Bogunovic and V. Kecojevic, "Artificial screen for reducing seismic vibration generated by blasting," Environmental Geology, vol. 53, no. 3, pp. 517-525, 2007.

[27] Y. F. Zhang, D. P. Yao, Z. Z. Xie, Y. X. Xu, G. M. Li, and Y. Q. Ye, "Analysis of master control factor of blasting seismic effect and discussion on shock absorption measures," Rock and Soil Mechanics, vol. 31, no. 1, pp. 304-308, 2010.

[28] D. Park, B. Jeon, and S. Jeon, "A numerical study on the screening of blast-induced waves for reducing ground vibration," Rock Mechanics and Rock Engineering, vol. 42, no. 3, pp. 449-473, 2009.

[29] G. Y. Gao, Z. Y. Li, C. Qiu, and Z. Q. Yue, “Three-dimensional analysis of rows of piles as passive barriers for ground vibration isolation," Soil Dynamics and Earthquake Engineering, vol. 26, no. 11, pp. 1015-1027, 2006.

[30] D. S. Kim and J. S. Lee, "Propagation and attenuation characteristics of various ground vibrations," Soil Dynamics and Earthquake Engineering, vol. 19, no. 2, pp. 115-126, 2000.

[31] J. Dai, Dynamic Behaviors and Blasting Theory of Rock, Metallurgical Industry Press, Beijing, China, 2013.

[32] W. H. Wang, X. B. Li, Z. L. Zhou, and Y. P. Zhang, "Energytransmitted rule of various stress waves across open joint," Journal of Central South University (Science and Technology), vol. 37, no. 2, pp. 376-380, 2006.

[33] L. L. Wang, Foundation of Stress Waves, National Defend Industry Press, 2005.
[34] Z. C. Zhang, Directional Pressure Relief Vibration Isolation Blasting, Chongqing University Press, Chongqing, China, 2013.

[35] X. B. Guo and J. C. Zhang, Blasting Engineering, China Communication Press, 2007.

[36] D. Y. Shi, Y. C. Li, and S. M. Zhang, The Analysis of Explicit Explosion Mechanics Base on Ansys/Ls-dyna8.1, Tsinghua University Press, Beijing, China, 2005.

[37] Livermore Software Technology Corporation, LS-DYNA Keyword User's Manual, Livermore Software Technology Corporation, Livermore, Calif, USA, 2007. 


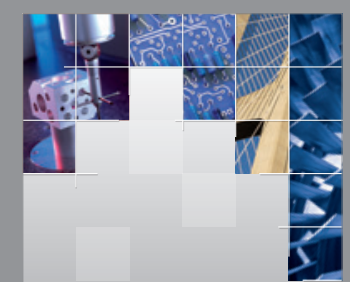

\section{Enfincering}
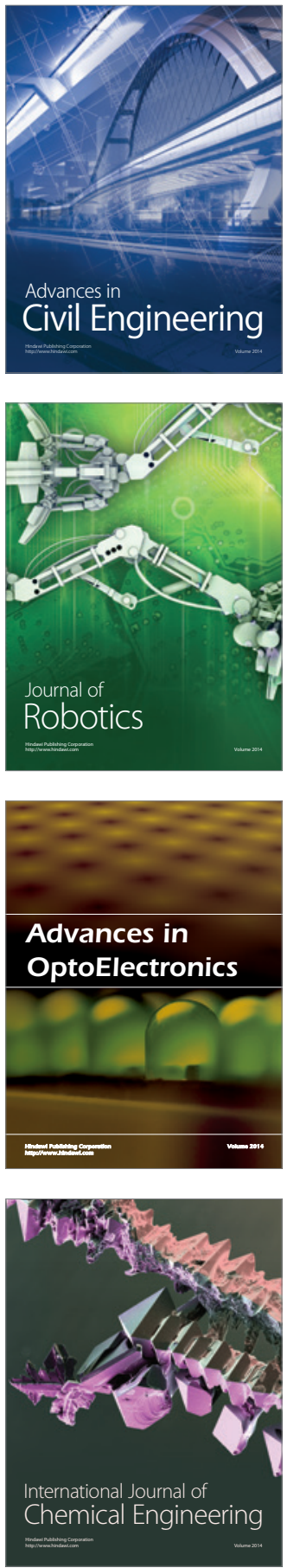

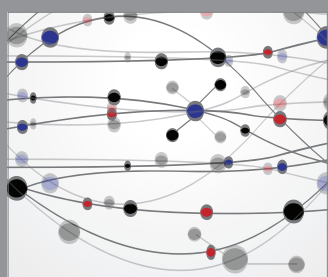

The Scientific World Journal

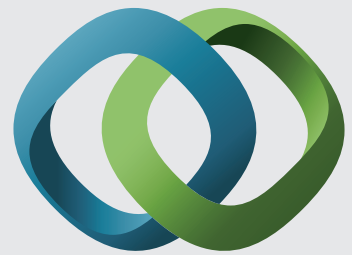

\section{Hindawi}

Submit your manuscripts at

http://www.hindawi.com
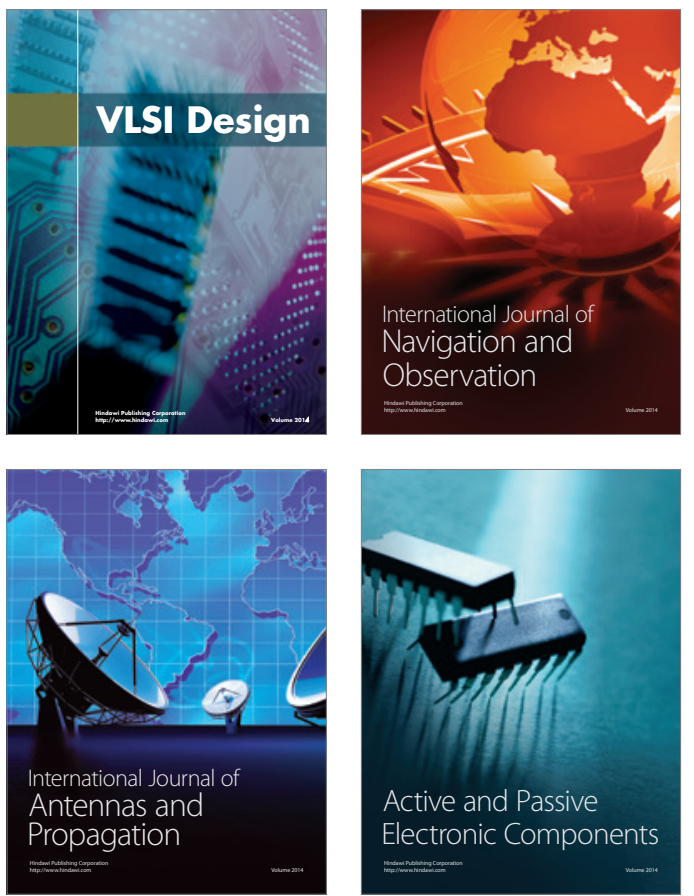
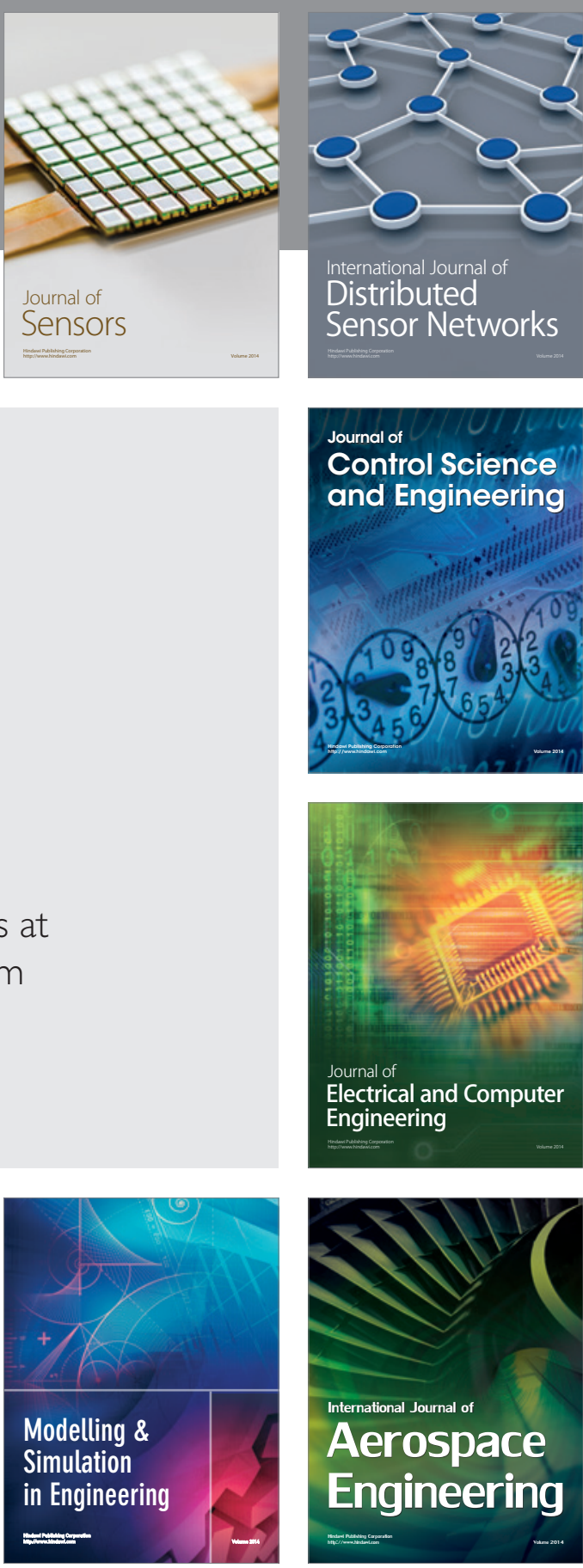

International Journal of

Distributed

Sensor Networks

Journal of

Control Science

and Engineering
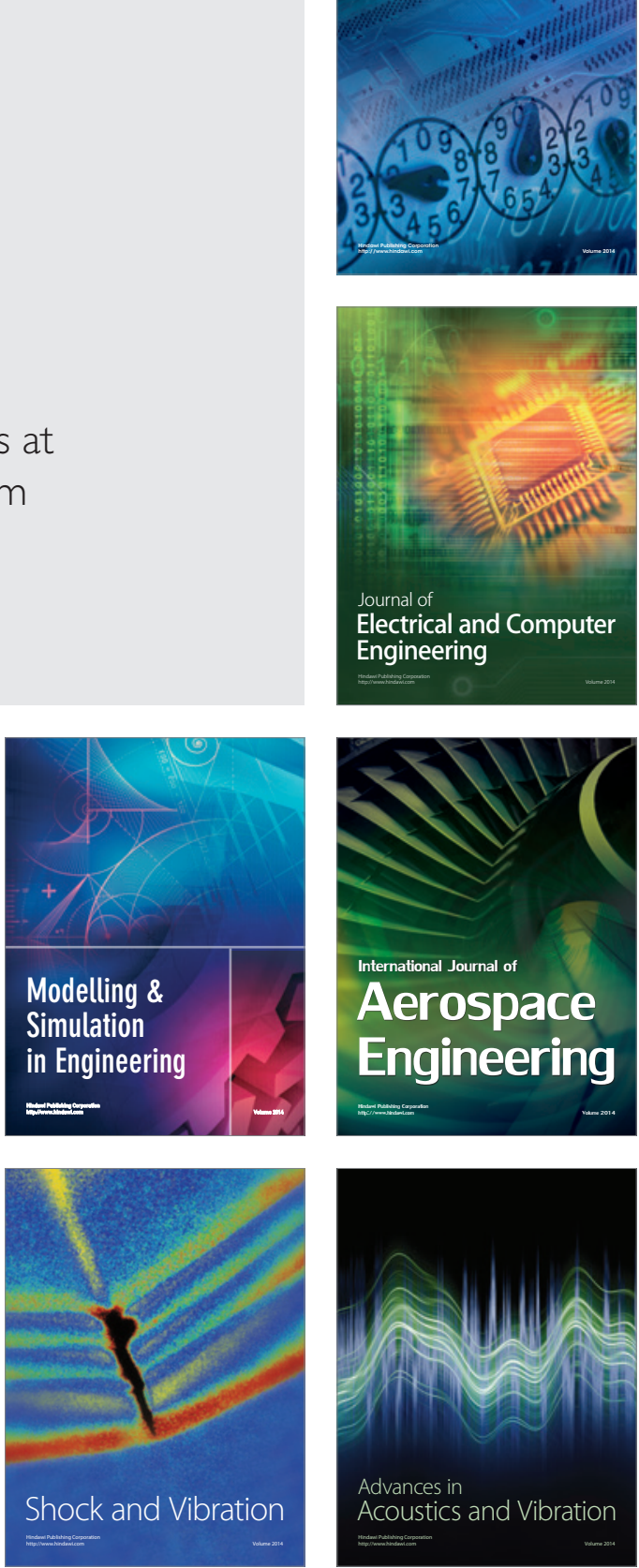